Research Article

\title{
Demand Side Management for Smart Grid Based on Smart Home Appliances with Renewable Energy Sources and an Energy Storage System
}

\author{
Zhihong Xu, ${ }^{1,2}$ Yan Gao ${ }^{D},{ }^{1}$ Muhammad Hussain, ${ }^{1}$ and Panhong Cheng ${ }^{1}$ \\ ${ }^{1}$ School of Management, University of Shanghai for Science and Technology, Shanghai 200093, China \\ ${ }^{2}$ Public Teaching Department, Rizhao Polytechnic, Rizhao 276826, Shandong, China \\ Correspondence should be addressed to Yan Gao; gaoyan@usst.edu.cn
}

Received 21 November 2019; Revised 1 March 2020; Accepted 17 March 2020; Published 27 April 2020

Academic Editor: Nazrul Islam

Copyright (c) 2020 Zhihong Xu et al. This is an open access article distributed under the Creative Commons Attribution License, which permits unrestricted use, distribution, and reproduction in any medium, provided the original work is properly cited.

A novel energy management system to improve the efficiency of renewable energy and storage system by scheduling various types of household appliances is developed. The end users schedule appliances optimally taking into account maximum utility (a measure of the satisfaction level of user's electricity consumption) as well as minimum user's cost of energy as competitive objectives. A random disturbance (reflecting an uncertainty) is introduced to describe the indeterminate amount of the electricity produced from the renewable energy sources at the adjacent slots. By applying the probability theory, the uncertain optimization model is transformed into a convex optimization problem. Then, the optimal solution is obtained using a quasi-Newton method. The rationality of our proposed model is verified through numerical simulations. According to the results of simulation studies, it is demonstrated that our proposed model not only enhances users' utility but also reduces energy consumption cost.

\section{Introduction}

1.1. Literature Review. The energy shortage and aggravation of environmental pollution are serious problems in a traditional power grid system. Power management is currently undergoing through a dramatic transformation from a traditional power system (TPS) into a smart grid [1]. The smart grid is an intellectualized power network system, which is based on highly integrated two-way communication networks and information systems. It is reliable, economical, and environmentally friendly and can be applied in sensing technologies and control infrastructures. Compared with traditional power grids, the smart grid has the advantages of flexible structure, excellent system performance, and high quality, which can improve the social development. Therefore, smart grid has become a beacon of development in the direction toward a modern power system [2-4].

Demand side management (DSM) in smart grid is a kind of management activity which can effectively promote and mobilize the enthusiasm of users to change or transfer the power consumption according to the dynamic prices (e.g., real-time pricing, time of use pricing, and inclining block rates) [5]. It is a hot topic for how to mobilize the enthusiasm of users to participate in their energy management. With improving the technology of renewable energy sources (RES) in recent years, many users are equipped with rooftop solar panels or small wind turbines. The RES is reducing the reliance on the TPS and relieving the supply pressure of power grids [6-9]. To alleviate the randomness and intermittent characteristics of the RES, an energy storage system (ESS) is being applied in smart grid, for example, popular electric vehicles that require both energy availability and storage power [10-13].

Scheduling the RES and ESS, power grids are becoming more secure and efficient in the electricity market $[10,14-22]$.

1.2. The Motivation of the Work and Our Contributions. Research on the management of RES and ESS are at their infancy [23-26]. Moreover, in current studies, RES and ESS have mainly focused on the foundation level of load 
prediction $[10,16,18,19,27-33]$. An optimization method [10] based on teaching-learning and adaptive neuro-fuzzy inference is used to estimate the maximum actual capacity of a charging state. A charging and discharging strategy of a future battery energy storage system was studied using an open distribution system simulator software to predict the daily load and photovoltaic (PV) generation curves [31]. The authors in [32], presented a simple method for determining the optimal capacity of PV power generation with a battery system based on the total annual solar radiation. The grid dependency can be obtained based on the total annual solar radiation without considering the time-series waveform of solar radiation. The studies need to know the production power distribution of the RES. However, in our model, a random disturbance (reflecting an uncertainty) is introduced to describe the indeterminate amount of the electricity produced from the RES at the adjacent slots. It is unnecessary to know about the distribution of electricity produced from the RES which is our first novelty.

Considering the RES or ESS, the classification of appliances is given in some studies $[16,34-42]$. A cost minimization problem focusing on the uninterruptible operation with an uncertainty of renewable energy generation at different time intervals was raised in [34]. In this study, uninterruptible and immovable electrical appliances were involved, whereas elastic appliances (such as air conditioners and water heaters) and semielastic ones (such as washing machines, dishwashers, and electric cookers) were not. A user convenience model employing a heuristic algorithm was proposed to minimize a user's dissatisfaction and the supplier's cost [36]. The model only involved appliances with beginning operation time and ending time. A robust optimization technique for scheduling smart appliances and electrical energy storage was applied to minimize the electricity bill and the $\mathrm{CO}_{2}$ emissions [37]. Chen et al. utilized a Lyapunov optimization approach to minimize the total payment from the grid and also have focused on semielastic appliances [38]. Similarly, authors in [39-42] elaborated on multilevel management with various RES and controllable loads, taking into account the energy saving and comfortable lifestyle as objectives of a realistic smart home energy management system.

Nevertheless, these works failed to consider the impacts of RES and ESS by scheduling different types of smart appliances, which is our second novelty.

To address these issues, an efficient model for demand side management is proposed, wherein, not only it is unnecessary to know about the distribution of electricity produced from the RES but also the impacts of RES and ESS are taken into account through managing various types of household appliances. In this way, users can optimally schedule operating set times of their smart home appliances and reasonably equip with the ESS and RES to achieve their energy management.

In our model, an expectation (caused by the uncertainty of RES) users' welfare maximization model is established. The users' utility maximization and bill minimization constitute the objective function. The users can design their electricity consumption mode according to the objective function. The constraint reflects the fluctuation (uncertainty) of electricity caused by the intermittency of the RES. The objective function is transformed into a deterministic problem and the constraint into the deterministic one. The model is then solved by applying the dual method. Finally, the rationality of the model and the validity of the algorithm are verified through numerical simulations. Our contributions are as follows:

(1) A random disturbance is introduced to describe the intermittence and instability of the RES at the adjacent slots. In this model, it is unnecessary to know about the distribution of electricity produced from the RES.

(2) An efficient model for demand side management is proposed so that users can reasonably arrange different smart appliances and consume the electricity supplied from the TPS, produced from the RES, or stored in the ESS concerning the users' utility maximization and their bill minimization.

The remainder of this paper is organized as follows. In Section 2, the classification of different appliances and the problem statement are formulated. We present the proposed model in Section 3. The results of numerical simulations are provided in Section 4, and some concluding remarks are given in Section 5.

The list of abbreviations is shown in Appendix. The architecture of the proposed system model in time slot $k$ is illustrated in Figure 1.

\section{Classification of Appliances and Problem Statement}

A system that includes a power supplier and multiple residential users is considered. The supplier and all users are linked using a communication infrastructure to realize information exchange. An energy hub (a detailed description given in [43]) of each user contains a rooftop solar panel, a small wind turbine, several batteries and electrical vehicles, some smart home appliances, and an electric quantity controller (EQC) with a smart meter. The smart meter not only bears a function of information transmission but also has the function of regulating and controlling household electricity information. It transmits the information of household electrical equipment consumption to users in order to better control the consumption and cost of electricity and sends relevant information to the power supplier to achieve load control and electricity price. The electricity price is given by the power supplier. Consumers can adjust the mode of electricity consumption by smart meters to achieve their maximum utility and minimum bill. In this model, it is supposed that users cannot sell redundant energy to the grid or other users, and thus the excess power from the RES is discarded [36].

Within the scope of our model, the devices' cost and the reactive power are not considered, and frequency and voltage stability are guaranteed [44]. The appliances are linked with the smart meter, where $t$ is the initial time, and 


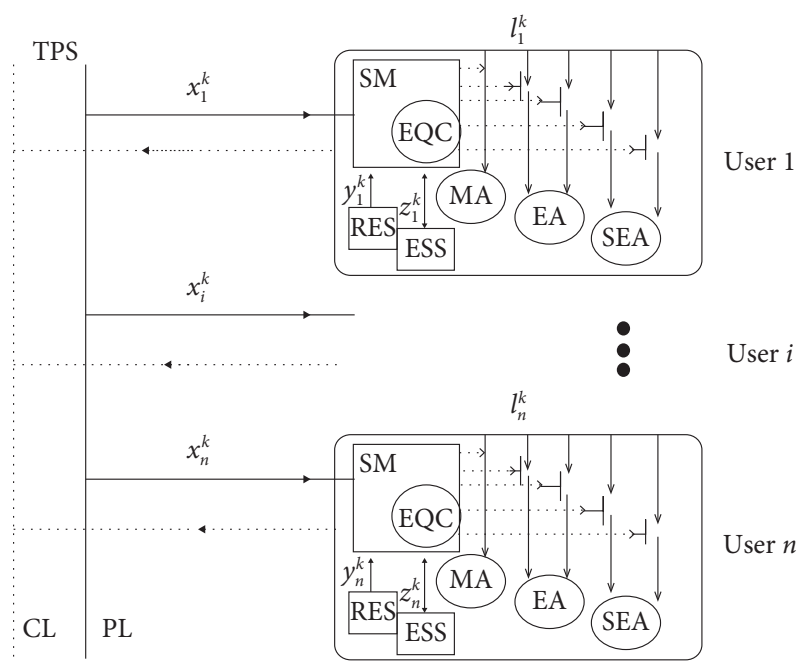

Figure 1: Architecture of the proposed system model in time slot $k$.

not is necessarily $1, K$ is the number of time slots, and $\mathbb{K}=$ $\{t, t+1, \ldots, K\}$ is the entire cycle. The indexes, functions and variables, and parameters are listed in Tables $1-3$, respectively. The modeling of the energy system components is detailed as follows.

2.1. Classification of Appliances. Every user has three types of appliances, namely, $A_{i}, B_{i}$, and $C_{i}$. The first type of household appliances, $A_{i}$, which are must-run appliances, are those household electrical appliances that must be operated. Owing to their inelasticity, this type of electrical appliances does not depend on the change of electricity price. For instance, lighting appliances, televisions, and refrigerators are not controlled by an EQC unit. The second category of household appliances, $B_{i}$, includes elastic equipment, such as air conditioners, water heaters, and other adjustable equipment. The use of these appliances is directly affected by the electricity price. When the electricity price rises, the electricity consumption decreases, and vice versa. The last type of household appliances, $C_{i}$, includes semielastic equipment, such as washing machines, dishwashers, electric cookers, and dryers. These appliances all require a certain amount of working time to complete an established task. Similar to elastic devices, semielastic devices are affected by the electricity price, whereas the working time of semielastic devices is fixed.

2.1.1. Must-Run Appliances. Let $l_{a^{k}}$ denote the power consumed by appliance $a_{u, i} \in A_{i} \cup B_{i}^{u, i} \cup C_{i}$ of user $i$ in time slot $k ; \mathbb{K}_{a_{u, i}}$ denotes the running time of a must-run appliance $a_{u, i} \in A_{i}$, which consumes $r_{a_{u, i}^{k}}$ in time slot $k$; and $\mathbb{K}_{a_{u, i}} \subseteq \mathbb{K}$. Denote $M_{A_{i}}$ as the number of must-run appliances, and let $E_{a_{u, i}}=\sum_{u=1}^{M_{A_{i}}} l_{a_{u, i}^{k}}$ denote the total power consumption of must-run appliances during an operation cycle.

2.1.2. Elastic Appliances. Denote $r_{a_{u, i}^{k}}^{\max }$ and $M_{B_{i}}$ as the maximum electricity consumed by elastic appliance $a_{u, i}$ and the number of elastic appliances, respectively, and
$R_{a_{u, i}^{k}}^{\max }=\sum_{u=1}^{M_{B_{i}}} r_{a_{u, i}^{k}}^{\max }$ denotes the maximum electricity consumed by all elastic appliances. The working models of these appliances are varied. The total electricity consumption $\sum_{u=1}^{M_{B_{i}}} l_{a_{u, i}^{k}}$ is given. Referring to this value, users can make their own unique electricity consumption mode. This means that, for the same total electricity consumption, the user has the opportunity to keep the water heater at a higher temperature during the winter or turn an electric fan on.

2.1.3. Semielastic Appliances. Semielastic appliances complete a task within a fixed time interval. Every household has $M_{C_{i}}$ semielastic appliances. Denote $E_{a_{u i}}^{\prime}$ as the total electricity of semielastic appliances. The working hours of these appliances are continuous. Once semielastic appliances are operated, they need to complete their tasks without stop. Here, $\mathbb{K}_{a_{u, i}}^{\prime}$ is the working time of semielastic appliances. The beginning time of an electrical appliance is $\alpha_{a j,}$, and the end time is $\beta_{a_{u i}}$. The working time can be expressed as $\left\{\alpha_{a_{u, i}}, \alpha_{a_{u, i}}+1, \ldots, \beta_{a_{u, i}}\right\} \in \mathbb{K}_{a_{u, i}^{\prime}}^{\prime}$. The satisfaction of the user will be seriously hindered if semielastic appliances are interrupted during an operation.

The three types of appliances satisfy the available electricity consumption, as indicated in Table 4 .

\subsection{Power Sources}

2.2.1. Traditional Power System. In this paper, TPS refers to the electricity supplied from the power supplier. Let $x_{i}^{k}$ denote the electricity supplied from a TPS in time slot $k$.

2.2.2. Renewable Energy Sources. The RES are deemed as significant generation alternatives in smart grid owing to their nonexhausted nature and benign environmental effects. Both a stochastic approach and a robust one are optimization methods for uncertain situations. The RES power generation is stochastic and intermittent and will be disturbed by many independent random factors. In the stochastic approach, the probability distribution of random parameters is known; in the robust one, the probability distribution of random parameters is unknown, but its fluctuation range needs to be known. In our model, unnecessary to know about the distribution of electricity produced from the RES, a random disturbance $\rho_{i}^{k}$ is introduced to describe the uncertainty of the RES with expectation value $E\left(\rho_{i}^{k}\right)=\mu_{i}^{k}$ and variance $D\left(\rho_{i}^{k}\right)=\left(\sigma_{i}^{k}\right)^{2}$. Then,

$$
y_{i}^{k+1}=y_{i}^{k}+\rho_{i}^{k+1}, \quad \forall k \in \mathbb{K},
$$

where $y_{i}^{k}$ denotes the electricity produced from the RES in time slot $k$.

By applying the Central Limit Theorem, when the number of users is large enough or segmentation of time slots is small enough, the sum of all random variables $\sum_{i=1}^{n} \sum_{j=t+1}^{k} \rho_{i}^{j}$ is subject to a normal distribution: 
TABle 1: List of indexes.

\begin{tabular}{|c|c|c|}
\hline Indexes & Description & Unit \\
\hline $\bar{t}$ & Initial time & $\mathrm{h}$ \\
\hline $\mathbb{K}$ & The entire cycle & $\mathrm{h}$ \\
\hline$k$ & Index of time slot & $\mathrm{h}$ \\
\hline$K$ & Number of time slots & $\mathrm{h}$ \\
\hline$i$ & Index of users & - \\
\hline$n$ & Number of users & - \\
\hline$A_{i}$ & Set of must-run appliances & - \\
\hline$B_{i}$ & Set of elastic appliances & - \\
\hline$C_{i}$ & Set of semielastic appliances & - \\
\hline$a_{u, i}$ & Electrical appliance & - \\
\hline$r_{a_{u, i}^{k}}$ & Electricity consumed by must-run appliance $a_{u, i}$ & $\mathrm{kWh}$ \\
\hline$K_{a_{u, i}}$ & Running time of must-run appliance $a_{u, i} \in A_{i}$ & $\mathrm{~h}$ \\
\hline$M_{A_{i}}$ & Number of must-run appliances $A_{i}$ & - \\
\hline$M_{B_{i}}$ & Number of elastic appliances $B_{i}$ & - \\
\hline$M_{C_{i}}$ & Number of semielastic appliances $C_{i}$ & - \\
\hline$E_{a_{u, i}}$ & Total electricity consumed by must-run appliance & $\mathrm{kWh}$ \\
\hline$r_{a_{u j}^{k}}^{\max }$ & Maximum electricity consumed by elastic appliance & $\mathrm{kWh}$ \\
\hline$R_{a_{i j}^{k}}^{\max _{i}}$ & Maximum total electricity consumed by elastic appliances & $\mathrm{kWh}$ \\
\hline$E_{a_{u, i}^{k}}^{u}$ & Total electricity of semielastic appliances & $\mathrm{kWh}$ \\
\hline$\alpha_{a_{u, i}}$ & Beginning time of semielastic appliances & $\mathrm{h}$ \\
\hline$\beta_{a_{u, i}}$ & End time of semielastic appliances & $\mathrm{h}$ \\
\hline $\mathbb{K}_{a_{u, i}^{\prime, i}}^{\prime}$ & Working time of semielastic appliances & $\mathrm{h}$ \\
\hline$C_{b}$ & Capacity of the ESS & $\mathrm{kWh}$ \\
\hline$g_{b}$ & Maximum value of the ESS & $\mathrm{kWh}$ \\
\hline$-g_{b}$ & Minimum value of the ESS & $\mathrm{kWh}$ \\
\hline$s_{i}^{0}$ & Initial charging state of the ESS & $\mathrm{kWh}$ \\
\hline$s_{i}^{k}$ & Charging state of the ESS & $\mathrm{kWh}$ \\
\hline$X$ & Set of electricity supplied from the TPS & $\mathrm{kWh}$ \\
\hline$Y$ & Set of electricity produced from the RES & $\mathrm{kWh}$ \\
\hline$Z$ & Set of electricity consumed in the ESS & $\mathrm{kWh}$ \\
\hline$G_{t}$ & Total electricity quantity of the power system & $\mathrm{kWh}$ \\
\hline
\end{tabular}

TABLE 2: List of functions and variables.

\begin{tabular}{lcc}
\hline Functions and variables & Description & Unit \\
\hline$x_{i}^{k}$ & Electricity supplied from the TPS & $\mathrm{kWh}$ \\
$y_{i}^{k}$ & Electricity produced from the RES & $\mathrm{kWh}$ \\
$z_{i}^{k}$ & Charging and discharging rates of the ESS & $\mathrm{kWh}$ \\
$U\left(l_{i}^{k}, \omega_{i}^{k}\right)$ & Utility function of user $i$ & $\$$ \\
$p_{k}$ & Price & Cents/kWh \\
$l_{i}^{k}$ & Total power consumed by user $i$ in time slot $k$ & $\mathrm{kWh}$ \\
$l_{a_{u, i}^{k}}^{k}$ & Electricity consumed by appliance $a_{u, i} \in A_{i} \cup B_{i} \cup C_{i}$ & $\mathrm{kWh}$ \\
$P\left(l_{i}^{k}\right)$ & Payment function of users & $\$$ \\
$\Phi(x)$ & Distribution function & - \\
\hline
\end{tabular}

TABLE 3: List of parameters.

\begin{tabular}{lcc}
\hline Parameters & Description & Unit \\
\hline$\alpha_{i}$ & Preset parameter & - \\
$\omega_{i}^{k}$ & Elastic coefficient & - \\
$m_{k}, n_{k}$ & Differentiated prices & Cents/kWh \\
$b_{k}$ & Energy consumption threshold in time slot $k$ & $\mathrm{kWh}$ \\
$\rho_{i}^{k}$ & Random disturbance to describe the indeterminate load produced by the RES & - \\
$\varepsilon, \eta$ & Positive numbers & - \\
$\lambda, \theta, \gamma$ & Lagrangian multipliers & - \\
\hline
\end{tabular}




$$
\sum_{i=1}^{n} \sum_{j=t+1}^{k} \rho_{i}^{j} \sim N\left(\mu, \sigma^{2}\right)
$$

where $N(\cdot)$ denotes a normal distribution function, $\mu=\sum_{i=1}^{n} \sum_{j=t+1}^{k} \mu_{i}^{j}$, and $\sigma^{2}=\sum_{i=1}^{n} \sum_{j=t+1}^{k}\left(\sigma_{i}^{j}\right)^{2}$.

Let $w=\left(-\sum_{i=1}^{n} \sum_{j=t+1}^{k} \rho_{i}^{j}+\mu\right) / \sigma$, the standardization of the normal distribution can be obtained as follows: $w \sim N(0,1)$; therefore, the distribution of all random variables is known why a stochastic approach is chosen instead of a robust one in our study.

2.2.3. Energy Storage System. Electric vehicles and batteries can be classified as the ESS, which can be charged or discharged. $C_{b}$ is denoted as the capacity of the ESS. In time slot $k$, the charge rate $z_{i}^{k}$ is positive and the discharge rate $z_{i}^{k}$ is negative. In addition, $g_{b}$ and $-g_{b}$ denote the maximum and minimum capacity values of the ESS, $-g_{b} \leq z_{i}^{k} \leq g_{b}, s_{i}^{k}$ is denoted as the capacity of the ESS at the beginning time slot $k$, and $s_{i}^{0}$ is the initial charging state of the ESS. At every time slot, the stored energy cannot exceed the storage capacity:

$$
s_{i}^{k}=s_{i}^{0}+\sum_{j=1}^{k-1} z_{i}^{j}, \quad 0 \leq s_{i}^{k} \leq C_{b} .
$$

2.3. Real-Time Pricing. The existing price forms mainly include fixed pricing, step pricing, time of use pricing (TOU), real-time pricing (RTP), and inclining block rates (IBR). Among them, RTP and IBR models have been extensively studied. It refers to the cost occurring at a certain moment during electricity sales, which reflects the characteristics of electricity prices with respect to time. Practically, power suppliers design the real-time price considering the time of demand and the power availability. Contrastingly in the IBR pricing model, as time goes on, prices remain unchanged, while incurring an increase when the energy consumption of a user reaches a predetermined threshold [19].

In our model, combining RTP and IBR price tariffs, the price $p_{k}$ is denoted as follows (see [45]):

$$
p_{k}\left(l_{i}^{k}\right)= \begin{cases}m_{k}, & 0 \leq l_{i}^{k} \leq b_{k}, \\ n_{k}, & l_{i}^{k}>b_{k},\end{cases}
$$

where $m_{k}$ and $n_{k}$ are differentiated prices, in which $m_{k} \leq n_{k}$; $l_{i}^{k}$ denotes the total electricity consumption of user $i$ in time slot $k$; and $b_{k}$ is the energy consumption threshold in time slot $k$.

2.4. Utility Function of a User. The concept of a utility function in microeconomics is widely used to characterize a user's satisfaction with a certain amount of electricity consumption. The existing studies have shown that the user's response can be expressed by a utility function. The electricity demand varies with each individual and depends upon the time of a day, climate, and price. The value of utility function denotes the satisfaction level of each user after using the electricity purchased. $U\left(l_{i}^{k}, \omega_{i}^{k}\right)$ is the utility function of user $i, l_{i}^{k}$ denotes the amount of consumed energy, and $\omega_{i}^{k}$ is an elastic coefficient and is a known parameter, and the utility value is expressed in a monetary form. Different utility functions represent different types of users and are distinguished by different elastic coefficients. Under the same conditions, the larger the $\omega_{i}^{k}$ value is, the higher the user' satisfaction. Usually, the general utility functions satisfy the following basic assumptions:

(1) The utility function is a nondecreasing function, i.e., $\partial U(l) / \partial l \geq 0$.

(2) Marginal utility is nonincreasing, i.e., the utility is a concave function. Thus, $\partial^{2} U(l) / \partial l^{2} \leq 0$.

(3) When the user does not consume electricity, the utility is zero, that is, $U(0)=0$.

In this paper, utility functions are considered as follows:

$$
U\left(l_{i}^{k}, \omega_{i}^{k}\right)= \begin{cases}\omega_{i}^{k} l_{i}^{k}-\frac{\alpha_{i}}{2}\left(l_{i}^{k}\right)^{2}, & 0 \leq l_{i}^{k} \leq \frac{\omega_{i}^{k}}{\alpha_{i}}, \\ \frac{\left(\omega_{i}^{k}\right)^{2}}{2 \alpha_{i}}, & l_{i}^{k}>\frac{\omega_{i}^{k}}{\alpha_{i}},\end{cases}
$$

where $\alpha_{i}$ is a preset parameter, and $\omega_{i}^{k}$ and $l_{i}^{k}$ are the elastic coefficient and power consumed by user $i$ in time slot $k$, respectively.

2.5. Payment Function of Users. The term $P\left(l_{i}^{k}\right)$ denotes a user's payment function. In our model, the devices' cost is not considered. Based on the price shown in (4), for a total load $l_{i}^{k}$ in time slot $k$, the user's cost $P\left(l_{i}^{k}\right)$ is determined as the maximum of two intersecting lines [45]:

$$
\begin{aligned}
& P\left(l_{i}^{k}\right)=p_{k} l_{i}^{k}=m_{k} l_{i}^{k}, \\
& P\left(l_{i}^{k}\right)=p_{k} l_{i}^{k}=n_{k} l_{i}^{k}+\left(m_{k}-n_{k}\right) b_{k} .
\end{aligned}
$$

The user's payment function is obtained from the following equation:

$$
P\left(l_{i}^{k}\right)=p_{k} l_{i}^{k}=\max \left\{m_{k} l_{i}^{k}, n_{k} l_{i}^{k}+\left(m_{k}-n_{k}\right) b_{k}\right\} .
$$

\section{Expectation Welfare Maximization Model}

3.1. Problem Formulation. In our model, based on scheduling household appliances and considering the fluctuation of electricity produced from the RES, an expectation model of users' welfare maximization is taken as follows:

(P1)

$$
\max E\left[\sum_{k=t}^{K} \sum_{i=1}^{n}\left(U\left(l_{i}^{k}, \omega_{i}^{k}\right)-P\left(l_{i}^{k}\right)\right)\right]
$$

subject to 


$$
\begin{aligned}
& 0 \leq l_{a_{u, i}^{k}} \leq r_{a_{u, i}^{k}}^{\max }, \quad \forall k \in \mathbb{K}, \forall a_{u, i} \in B_{i}, \\
& 0 \leq l_{a_{u, i}^{k}} \leq r_{a_{u, i}^{k}}^{\max }, \quad \forall k \in \mathbb{K}_{a_{u, i}}^{\prime}, \forall a_{u, i} \in C_{i}, \\
& l_{a_{u, i}^{k}}=0, \quad \forall k \notin \mathbb{K}_{a_{u, i}}^{\prime}, \forall a_{u, i} \in C_{i}, \\
& \sum_{k \in \mathbb{K}_{a_{u, i}}^{\prime}} l_{a_{u, i}^{k}}=E_{a_{u, i}}^{\prime}, \quad \forall a_{u, i} \in C_{i}, \\
& l_{i}^{k}=E_{a_{u, i}^{k}}+\sum_{a_{u, i} \in B_{i}} l_{a_{u, i}^{k}}+\sum_{a_{u, i} \in C_{i}} l_{a_{u, i}^{k}}, \quad \forall k \in \mathbb{K}, \\
& y_{i}^{k+1}=y_{i}^{k}+\rho_{i}^{k+1}, \quad \forall k \in \mathbb{K}, \\
& z_{i}^{k} \in\left[-g_{b}, g_{b}\right], \\
& s_{i}^{k}=s_{i}^{0}+\sum_{j=1}^{k-1} z_{i}^{j}, \quad 0 \leq s_{i}^{k} \leq C_{b}, \forall k \in \mathbb{K}, \\
& l_{i}^{k}=\left(x_{i}^{k}-y_{i}^{k}+z_{i}^{k}\right)^{+},
\end{aligned}
$$

where $U\left(l_{i}^{k}, \omega_{i}^{k}\right)$ and $P\left(l_{i}^{k}\right)$ are the utility function and payment function of user $i$ in time slot $k$, respectively, $\omega_{i}^{k}$ (reflecting the satisfaction level of each user after using the electricity purchased) is an elastic coefficient and is a known parameter, $x_{i}^{k}$ is the amount of electricity supplied from the TPS, $y_{i}^{k}$ is the amount of electricity produced from the RES, and $z_{i}^{k}$ is the electricity charged or discharged in the ESS. In addition, $x_{i}^{k}, y_{i}^{k}$, and $z_{i}^{k}$ are variables which the amount is eight hundred and forty and $G_{k}$ is the total electricity quantity of a power system.
The constraints (9a) and (9b)-(9d) characterize the electric performance of elastic and semielastic appliances, respectively. The compositions and sources of a user's electricity consumption are expressed as constraints (9e) and (9i). Constraint (9f) is the power fluctuation generated from the RES between two time slots. This constraint on the RES is not considered in [46]. Besides, determining the relationship between $y_{i}^{k}$ and $y_{i}^{t}, y_{i}^{k}$ can be expressed as follows:

$$
y_{i}^{k}=y_{i}^{t}+\sum_{j=t+1}^{k} \rho_{i}^{j} .
$$

Constraint (9g) describes the electric performance of the ESS. Constraint $(9 \mathrm{~h})$ shows that the expected total electricity consumption of users does not exceed the electricity production, which reflects the correlation and coupling between the electricity consumption of users. Samadi et al. do not take the constraint into account [35].

3.2. Convex Optimization Problem Formulation. The transformation of the objective function and constraint (9h) are shown as Appendixes A and B.

According to Appendixes A and B, a convex optimization problem from problem (P1) is obtained as

(P2)

$$
\max \sum_{k=t}^{K} \sum_{i=1}^{n}\left[\widetilde{U}\left(l_{i}^{t}, \omega_{i}^{k}\right)-\widetilde{P}\left(l_{i}^{k}\right)\right],
$$

subject to $(9 \mathrm{a})-(9 \mathrm{e}),(10 \mathrm{a}),(9 \mathrm{~g})$, and (9i)

$$
\begin{aligned}
& \sum_{i=1}^{n}\left(x_{i}^{t}-y_{i}^{t}+z_{i}^{t}\right)^{+} \leq G_{t}, \quad k=t, \\
& \sum_{i=1}^{n}\left(x_{i}^{t}-y_{i}^{t}+z_{i}^{k}\right)^{+} \leq G_{k}+\varphi(k), \quad k=t+1, \ldots, K,
\end{aligned}
$$

in which,

$$
\begin{aligned}
& \tilde{U}_{i}\left(l_{i}^{t}, \omega_{i}^{k}\right)=\left\{\begin{array}{l}
\omega_{i}^{k} l_{i}^{t}-\frac{\alpha_{i}}{2}\left(l_{i}^{t}\right)^{2}-\omega_{i}^{k} \sum_{j=t+1}^{k} \mu_{i}^{j}+\alpha_{i} l_{i}^{t} \sum_{j=t+1}^{k} \mu_{i}^{j}-\frac{\alpha_{i}}{2}\left(\sum_{j=t+1}^{k} \mu_{i}^{j}\right)^{2}-\frac{\alpha_{i}}{2}\left(\sum_{j=t+1}^{k} \sigma_{i}^{j}\right)^{2}, \quad 0 \leq l_{i}^{t} \leq \frac{\omega_{i}^{k}}{\alpha_{i}}, \\
\frac{\left(\omega_{i}^{k}\right)^{2}}{2 \alpha_{i}}-\omega_{i}^{k} \sum_{j=t+1}^{k} \mu_{i}^{j}+\alpha_{i} l_{i}^{t} \sum_{j=t+1}^{k} \mu_{i}^{j}-\frac{\alpha_{i}}{2}\left(\sum_{j=t+1}^{k} \mu_{i}^{j}\right)^{2}-\frac{\alpha_{i}}{2}\left(\sum_{j=t+1}^{k} \sigma_{i}^{j}\right)^{2},
\end{array}\right. \\
& \widetilde{P}\left(l_{i}^{k}\right)=\max \left\{m_{k}\left(l_{i}^{t}-\sum_{j=t+1}^{k} \mu_{i}^{j}\right)^{+}, n_{k}\left(l_{i}^{t}-\sum_{j=t+1}^{k} \mu_{i}^{j}\right)^{+}+\left(m_{k}-n_{k}\right) b_{k}\right\},
\end{aligned}
$$

$E\left(\rho_{i}^{k}\right)=\mu_{i}^{k}$ and $D\left(\rho_{i}^{k}\right)=\left(\sigma_{i}^{k}\right)^{2}$ are the expectation and variance of the random disturbance $\rho_{i}^{k}$, respectively.

Among these constraints, constraints (9g), (12a), and (12b) are our inequality constraints, and constraints (9d) are our equality ones. The type of decision variables is real, and there are eight hundred and forty variables, one hundred and thirty-nine inequality constraints, twenty equality constraints, and eight hundred and forty boundaries. 
Theorem 1. Problem (P2) is a convex optimization and has an optimal solution.

Proof of Theorem 1 is shown in Appendix C.
3.3. Dual Problem. From Theorem 1, (P2) is a convex optimization and has an optimal solution. It is equivalent to its dual problem. Therefore, it can be solved using a dual method [20].

The Lagrangian function of (P2) is

$$
\begin{aligned}
L(X, Y, Z, \lambda, \theta, \gamma)= & \sum_{k=t}^{K} \sum_{i=1}^{n}\left(\widetilde{U}\left(l_{i}^{t}, \omega_{i}^{k}\right)-\widetilde{P}\left(l_{i}^{k}\right)\right)-\lambda\left(\sum_{i=1}^{n}\left(x_{i}^{t}-y_{i}^{t}+z_{i}^{t}\right)^{+}-G_{t}+\sum_{k=t+1}^{K}\left(\sum_{i=1}^{n}\left(x_{i}^{t}-y_{i}^{t}+z_{i}^{k}\right)^{+}-G_{k}-\varphi(k)\right)\right) \\
& -\sum_{i=1}^{n} \sum_{a_{u, i} \in C_{i}} \theta_{a_{u, i}}\left(E_{a_{u, i}{ }^{\prime}}-\sum_{k \in K_{a_{u, i}}} l_{a_{u, i}^{k}}\right)-\gamma \sum_{k=t}^{K} \sum_{i=1}^{n}\left(s_{i}^{k}-C_{b}\right) \\
= & \sum_{i=1}^{n} \sum_{k=t}^{K}\left(\widetilde{U}\left(l_{i}^{t}, \omega_{i}^{k}\right)-\widetilde{P}\left(l_{i}^{k}\right)\right)-\sum_{i=1}^{n} \sum_{k=t}^{K} \lambda\left(x_{i}^{t}-y_{i}^{t}+z_{i}^{k}\right)^{+}+\lambda \sum_{k=t}^{K} G_{k}+\lambda \sum_{k=t+1}^{K} \varphi(k)-\sum_{i=1}^{n} \sum_{a_{u, i} \in C_{i}} \theta_{a_{u, i}}\left(E_{a_{u, i}{ }^{\prime}}-\sum_{k \in K_{a_{u, i}}{ }^{\prime}} l_{a_{u, i}^{k}}\right) \\
& -\gamma \sum_{k=t}^{K} \sum_{i=1}^{n}\left(s_{i}^{k}-C_{b}\right),
\end{aligned}
$$

where $\quad X=\left(x_{1}^{t}, x_{2}^{t}, \ldots, x_{n}^{t}\right), \quad Y=\left(y_{1}^{t}, y_{2}^{t}, \ldots, y_{n}^{t}\right)$, $Z=\left(z_{1}^{t}, z_{2}^{t}, \ldots, z_{n}^{t}\right), \quad \lambda \geq 0, \quad \theta=\left(\theta_{a_{u, i}}\right)_{M_{C_{i}} \times n} \in R^{M_{C_{i}} \times n}$, and $\gamma \in R^{+}$. The objective function of the dual optimization problem is as follows:

$$
D(\lambda, \theta, \gamma)=\max _{x_{i}^{k}, y_{i}^{k}, z_{i}^{k}}\left\{\begin{array}{l|l}
L(X, Y, Z, \lambda, \theta, \gamma) & \begin{array}{l}
0 \leq l_{a_{u, i}^{k}} \leq r_{a_{u, i}^{k}}^{\max }, \forall a_{u, i} \in B_{i}, C_{i}, \\
z_{i}^{k} \in\left[-g_{b}, g_{b}\right], \forall k \in K, \\
l_{i}^{k}=\left(x_{i}^{k}-y_{i}^{k}+z_{i}^{k}\right)^{+} .
\end{array}
\end{array}\right\}
$$

The dual problem is the following:

$$
\min _{\lambda, \theta, \gamma \in R^{+}} D(\lambda, \theta, \gamma)
$$

\subsection{Smoothing Method}

3.4.1. Introduction of Smoothing Methods. The payment function $\widetilde{P}\left(l_{i}^{k}\right)$ of (P2) contains of the plus and maximum functions, which are nonsmooth. It is impossible to solve this problem directly using classical optimization methods. Smooth approximation functions can be found to replace the abovementioned functions. The basic idea of a smoothing method is to replace the nonsmooth function with a smooth approximation function and solving the original problem is equivalent to solving the traditional optimization problem. There are many smoothing methods, such as quadratic function method, aggregate function method, and density method (see [47]).

Absolute value function $y=|x|$ is convex on $R^{1}$ and smooth everywhere except $x=0$. Given a small constant $\varepsilon>0$, the following smooth function approximates it:

$$
f_{\varepsilon}(x)= \begin{cases}-x, & x \leq-\varepsilon, \\ \frac{1}{2 \varepsilon} x^{2}+\frac{\varepsilon}{2}, & -\varepsilon<x<\varepsilon, \\ x, & x \geq \varepsilon,\end{cases}
$$

which is called quadratic function method.

The aggregate function method is considered. The maximum function is as follows:

$$
f(x)=\max _{1 \leq i \leq m} f_{i}(x),
$$

where $f_{i}(x)(i=1, \ldots, m)$ is a second-order continuous differentiable function on $R^{n}$. The following aggregate function is a smooth approximation function of formula (18) [47]:

$$
f(x, \pi)=\pi \ln \left[\sum_{i=1}^{m} \exp \left(\frac{f_{i}(x)}{\pi}\right)\right],
$$

where $\pi$ is a small positive number.

Next, the density method is discussed.

Let $\rho(x)$ be a probability density function from $R^{1}$ to $R^{+}$ and satisfy 


$$
\int_{-\infty}^{+\infty}|s| \rho(s) \mathrm{d} s=\kappa<+! \infty
$$

The smooth approximation function of $x_{+}=\max \{x, 0\}$ is as follows:

$$
P(\mu, x)=\int_{-\infty}^{+\infty}(x-\mu s)_{+} \rho(s) \mathrm{d} s,
$$

where $\mu>0$ is a smaller number.

Because the plus function is inside and the maximum function is outside of the payment function $\widetilde{P}\left(l_{i}^{k}\right)$, the plus function is considered first smoothing and then smoothing the maximum function.

3.4.2. First Smoothing. The quadratic function and aggregate function method are simple, practical, and effective, and these smoothing methods are adopted to deal with the nonsmoothness of payment function $\widetilde{P}\left(l_{i}^{k}\right)$. The quadratic method is first adopted.
When $x=0, \psi(x)=\max \{x, 0\}$ is nonsmooth, and let

$$
\psi_{t}(x)= \begin{cases}x, & x>t \\ \frac{x^{2}}{4 t}+\frac{x}{2}+\frac{t}{4}, & -t \leq x \leq t \\ 0, & x<-t .\end{cases}
$$

Theorem 2. For $\forall t>0, \psi_{t}(x)$, in formula (22) is a smooth approximation function of $\psi(x)=\max \{x, 0\}$.

Proof of Theorem 2 is shown as Appendix D.

According to Theorem 2, $x$ of formula (22) is directly replaced by $\left(l_{i_{j}}^{t}-\sum_{j=t+1}^{k} \mu_{i}^{j}\right)^{+}$and the smoothing function $\psi_{t}\left(l_{i}^{t}-\sum_{j=t+1}^{k} \mu_{i}^{j}\right)$ can be obtained. Then,

$$
\begin{aligned}
\widetilde{P}\left(l_{i}^{k}\right) & =\max \left\{m_{k}\left(l_{i}^{t}-\sum_{j=t+1}^{k} \mu_{i}^{j}\right)^{+}, n_{k}\left(l_{i}^{t}-\sum_{j=t+1}^{k} \mu_{i}^{j}\right)^{+}+\left(m_{k}-n_{k}\right) b_{k}\right\} \\
& =\max \left\{m_{k} \psi_{t}\left(l_{i}^{t}-\sum_{j=t+1}^{k} \mu_{i}^{j}\right), n_{k} \psi_{t}\left(l_{i}^{t}-\sum_{j=t+1}^{k} \mu_{i}^{j}\right)+\left(m_{k}-n_{k}\right) b_{k}\right\} .
\end{aligned}
$$

3.4.3. Second Smoothing. During the second smoothing process, the aggregate function method is used to smooth the maximum function of the payment function $\widetilde{P}\left(l_{i}^{k}\right)$.

Let $\widetilde{P}\left(l_{i}^{k}\right)=\max \left\{f_{1}(x), f_{2}(x)\right\}$, where $f_{1}(x)=m_{k} \psi_{t}$ $\left(l_{i}^{t}-\sum_{j=t+1}^{k} \mu_{i}^{j}\right)$ and $f_{2}(x)=n_{k} \psi_{t}\left(l_{i}^{t}-\sum_{j=t+1}^{k} \mu_{i}^{j}\right)+\left(m_{k}-\right.$ $\left.n_{k}\right) b_{k}$.
Thus, $L(X, Y, Z, \lambda, \theta, \gamma)$ is smoothed to

$$
\begin{aligned}
L_{t u}(X, Y, Z, \lambda, \theta, \gamma)= & \sum_{k=t}^{K} \sum_{i=1}^{n}\left(\widetilde{U}\left(l_{i}^{t}, \omega_{i}^{k}\right)-\widetilde{P}\left(l_{i}^{k}\right)_{u}\right)-\lambda\left(\sum_{i=1}^{n}\left(x_{i}^{t}-y_{i}^{t}+z_{i}^{t}\right)^{+}-G_{t}+\sum_{k=t+1}^{K}\left(\sum_{i=1}^{n}\left(x_{i}^{t}-y_{i}^{t}+z_{i}^{k}\right)^{+}-G_{k}-\varphi(k)\right)\right) \\
& -\sum_{i=1}^{n} \sum_{a_{u, i} \in C_{i}} \theta_{a_{u, i}}\left(E_{a_{u, i}^{k}}^{\prime}-\sum_{k \in K_{a_{u, i}}} l_{a_{u, i}^{k}}\right)-\gamma \sum_{k=t}^{K} \sum_{i=1}^{n}\left(s_{i}^{k}-C_{b}\right),
\end{aligned}
$$

and the model in (15) can be transformed to find the maximum value of the smoothing approximation function:

$$
D(\lambda, \theta, \gamma)=\max _{x_{i}^{k}, y_{i}^{k}, z_{i}^{k}}\left\{\begin{array}{l|l}
L_{t u}(X, Y, Z, \lambda, \theta, \gamma) & \begin{array}{l}
0 \leq l_{a_{u, i}^{k}} \leq r_{a_{u, i}^{k}}^{\max }, \forall a_{u, i} \in B_{i}, C_{i}, \\
z_{i}^{k} \in\left[-g_{b}, g_{b}\right], \quad \forall k \in K, \\
l_{i}^{k}=\left(x_{i}^{k}-y_{i}^{k}+z_{i}^{k}\right)^{+} .
\end{array}
\end{array}\right\}
$$

The optimal solution of (25) can be obtained using a quasi-Newton method [34]:
(1) Taking $X_{0}, H_{0}$, and parameter $\varepsilon \geq 0$, let $k=0$. 
TABle 4: Available electricity consumption of appliances.

\begin{tabular}{|c|c|c|}
\hline Appliances & Parameters & Available electricity consumption \\
\hline Must-run appliances $A_{i}$ & $\mathbb{K}_{a_{u, i}}, r_{a_{u, i}^{k}}$ & $l_{a_{u, i}^{k}}= \begin{cases}r_{a_{u, i}^{k}}, & k \in \mathbb{K}_{a_{u, i}} \\
0, & \text { otherwise }\end{cases}$ \\
\hline Elastic appliances $B_{i}$ & $r_{a_{u, i}^{k}}^{\max }$ & $0 \leq l_{a_{u, i}^{k}} \leq r_{a_{u, i}^{k}}^{\max }, \forall k \in \mathbb{K}$ \\
\hline Semielastic appliances $C_{i}$ & $r_{a_{u, i}^{k}}^{\max }, \mathbb{K}_{a_{u, i}}^{\prime}, E_{a_{u, i}}^{\prime}$ & $\begin{array}{c}0 \leq l_{a_{u, i}^{k}} \leq r_{a_{u, i}^{k}}^{\max }, \forall k \in \mathbb{K}_{a_{u, i}}^{\prime} \\
l_{a_{u, i}^{k}}=0, \forall k \notin \mathbb{K}_{a_{u, i}^{\prime}}^{\prime} \\
\sum_{k \in \mathbb{K}_{a_{u, i}}^{\prime}} l_{a_{u, i}^{k}}=E_{a_{u, i}}^{\prime}\end{array}$ \\
\hline
\end{tabular}

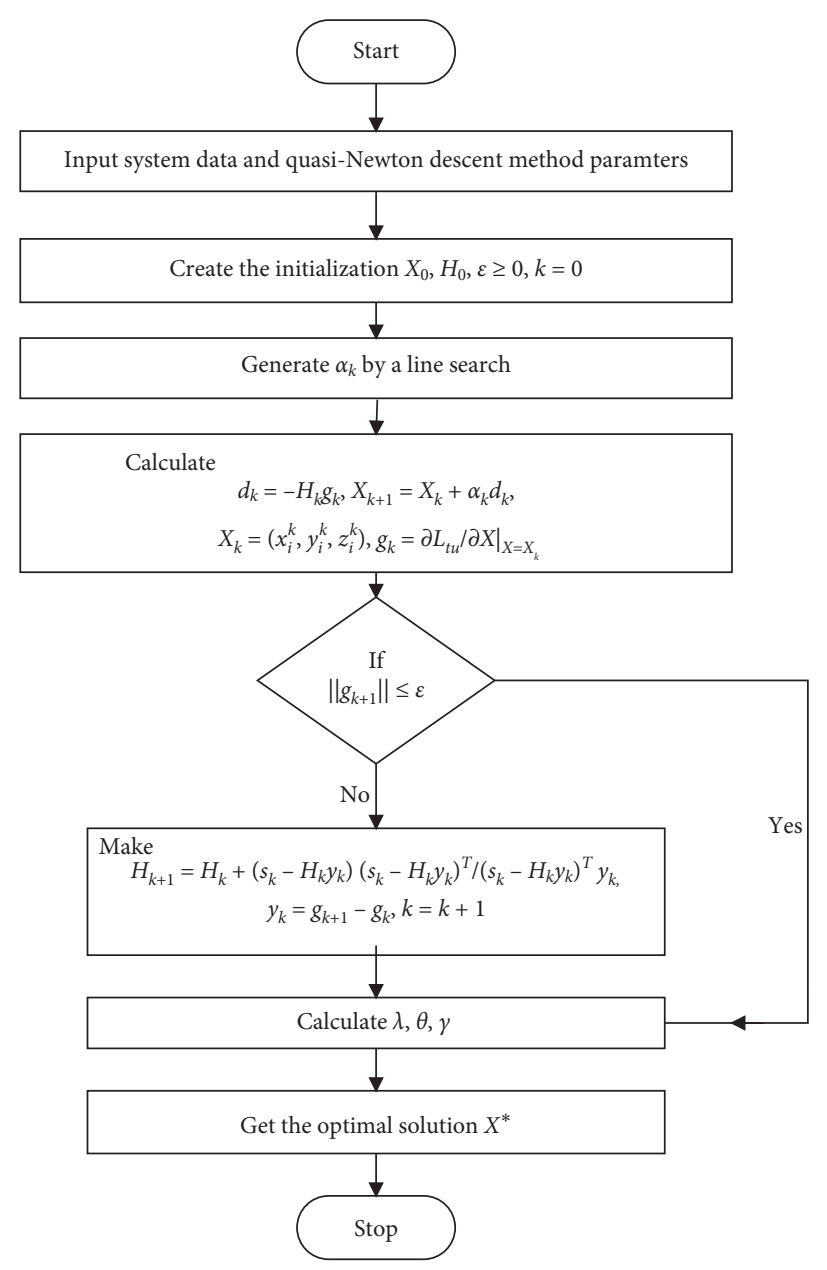

FIgUre 2: A flowchart of the algorithm.

(2) Computing $d_{k}=-H_{k} g_{k}$ and $X_{k+1}=X_{k}+\alpha_{k} d_{k}$, where $X_{k}=\left(x_{i}^{k}, y_{i}^{k}, z_{i}^{k}\right), g_{k}=\left.\left(\partial L_{t u} / \partial X\right)\right|_{X=X_{k}}, \alpha_{k}$ is a step generated by a line search.

If $\left\|g_{k+1}\right\| \leq \varepsilon$, the algorithm stops; otherwise, moves to the next step.

(3) Let $H_{k+1}=H_{k}+\left(\left(s_{k}-H_{k} y_{k}\right)\left(s_{k}-H_{k} y_{k}\right)^{T}\right) /\left(\left(s_{k}-\right.\right.$ $\left.\left.H_{k} y_{k}\right)^{T} y_{k}\right)$, where $y_{k}=g_{k+1}-g_{k}$, and $k=k+1$, go to $(2)$.

Thus, $X^{*}(\lambda, \theta, \gamma)$ is obtained and is substituted into (25). We can then have $D(\lambda, \theta, \gamma)$. Computing $\lambda, \theta$, and $\gamma$, the solution of the model is obtained.

A flowchart of the algorithm is shown in Figure 2.

\section{Numerical Simulations}

A power system that includes a power supplier with five users is considered. One day is divided into twenty-four periods. Each family is equipped with a rooftop solar panel, a small wind turbine, several batteries and electrical vehicles, and ten electric appliances. The must-run appliances $a_{1}$, $a_{2} \in A_{i}$ consume electricity at any time during the day. The electricity consumption of these appliances is set as $1 \mathrm{kWh}$ and $3 \mathrm{kWh}$. The elastic appliances $B_{i}$, include four appliances $a_{3}, a_{4}, a_{5}$, and $a_{6}$. Their electricity consumption is considered as a whole, so long as the total electricity consumption will not exceed $R_{a_{u, i}^{k}}^{\max }=\sum_{a_{u, i} \in B_{i}} r_{a_{u, i}^{k}}^{\max }=10 \mathrm{kWh}$. The 
TABLE 5: The parameters of appliances.

\begin{tabular}{|c|c|c|c|c|c|c|c|c|c|c|}
\hline \multirow{3}{*}{$\begin{array}{l}\text { The types of Appliances } \\
\text { Appliance } \\
\text { Electricity (kWh) } \\
\text { Schedule time frame (h) }\end{array}$} & \multicolumn{2}{|c|}{$\begin{array}{c}\text { Must-run } \\
\text { appliances } A_{i}\end{array}$} & \multicolumn{4}{|c|}{$\begin{array}{c}\text { Elasti } \\
\text { c appliances } B_{i}\end{array}$} & \multicolumn{4}{|c|}{$\begin{array}{c}\text { Semielastic } \\
\text { appliances } C_{i}\end{array}$} \\
\hline & $a_{1}$ & $a_{2}$ & $a_{3}$ & $a_{4}$ & $a_{5}$ & $a_{6}$ & $\begin{array}{c}a_{7} \\
5\end{array}$ & $\begin{array}{c}a_{8} \\
3\end{array}$ & $\begin{array}{c}a_{9} \\
4\end{array}$ & $\begin{array}{c}a_{10} \\
2\end{array}$ \\
\hline & - & - & - & - & - & - & $3 \mathrm{am}-9 \mathrm{am}$ & $5 \mathrm{am}-11 \mathrm{am}$ & 4 am-2 pm & $9 \mathrm{am}-6 \mathrm{pm}$ \\
\hline
\end{tabular}

TABLE 6: The indexes used in the models.

\begin{tabular}{|c|c|c|c|c|}
\hline Indexes & $w=2$ & $w=6$ & $w=10$ & $w=20$ \\
\hline The intervals of $\omega_{i}^{k}$ & {$[1,3]$} & {$[5,7]$} & {$[9,11]$} & {$[19,21]$} \\
\hline The satisfaction levels of users & Lowest & & & Highest \\
\hline
\end{tabular}

semielastic appliances, include four appliances $a_{7}, a_{8}, a_{9}$, and $a_{10}$. The total amount of electricity required for their tasks is $5 \mathrm{kWh}, 3 \mathrm{kWh}, 4 \mathrm{kWh}$, and $2 \mathrm{kWh}$, respectively, and the expected working periods are $\{3,4, \ldots, 9\},\{5,6, \ldots, 11\},\{4$, $5, \ldots, 14\}$, and $\{9,10, \ldots, 18\}$. The capacities of the RES and ESS are set as $20 \mathrm{kWh}$ and $5 \mathrm{kWh}$, respectively. All the computations are performed by Matlab R2016a. The running time for all the algorithm simulations is less than $1 \mathrm{~ms}$. The abovementioned data are shown in Table 5.

The differentiated prices $m_{k}$ and $n_{k}$ are set as follows:

$$
\begin{gathered}
m_{k}=\left\{\begin{array}{l}
3 \text { cents/kWh, [0: 00, 10: 00], } \\
7 \text { cents/kWh, [10: 00, 16: 00], } \\
10 \text { cents/kWh, [16: 00, 20: 00], } \\
8 \text { cents/kWh, [20: 00, 24:00], }
\end{array}\right. \\
n_{k}=\left\{\begin{array}{l}
9 \text { cents/kWh, [0: 00, 8: 00], } \\
7 \text { cents/kWh, [8: 00, 13:00], } \\
15 \text { cents/kWh, [13: 00,24:00]. }
\end{array}\right.
\end{gathered}
$$

Let $b_{k}=4 \mathrm{~kW}$ during the entire period.

The utility function of user $i$ is set as follows:

$$
U\left(l_{i}^{k}, \omega_{i}^{k}\right)= \begin{cases}\omega_{i}^{k} l_{i}^{k}-\frac{\alpha_{i}}{2}\left(l_{i}^{k}\right)^{2}, & 0 \leq l_{i}^{k} \leq \frac{\omega_{i}^{k}}{\alpha_{i}} \\ \frac{\left(\omega_{i}^{k}\right)^{2}}{2 \alpha_{i}}, & l_{i}^{k}>\frac{\omega_{i}^{k}}{\alpha_{i}}\end{cases}
$$

where $\omega_{i}^{k}$ is an elastic utility coefficient and is a known parameter. Under the same conditions, the larger is the value of $\omega_{i}^{k}$, the higher is the power consumption while increasing the pressure on the TPS (further information regarding the utility function can be found in [48]). Therefore, $\omega_{i}^{k}$ needs to be taken into account as a proper range. In the first scenario, different ranges of the elastic coefficient $\omega_{i}^{k}$ are analyzed. In the other scenario, the impacts of the RES and ESS by manipulating semielastic and elastic appliances are studied under the appropriate range of $\omega_{i}^{k}$ in various models.

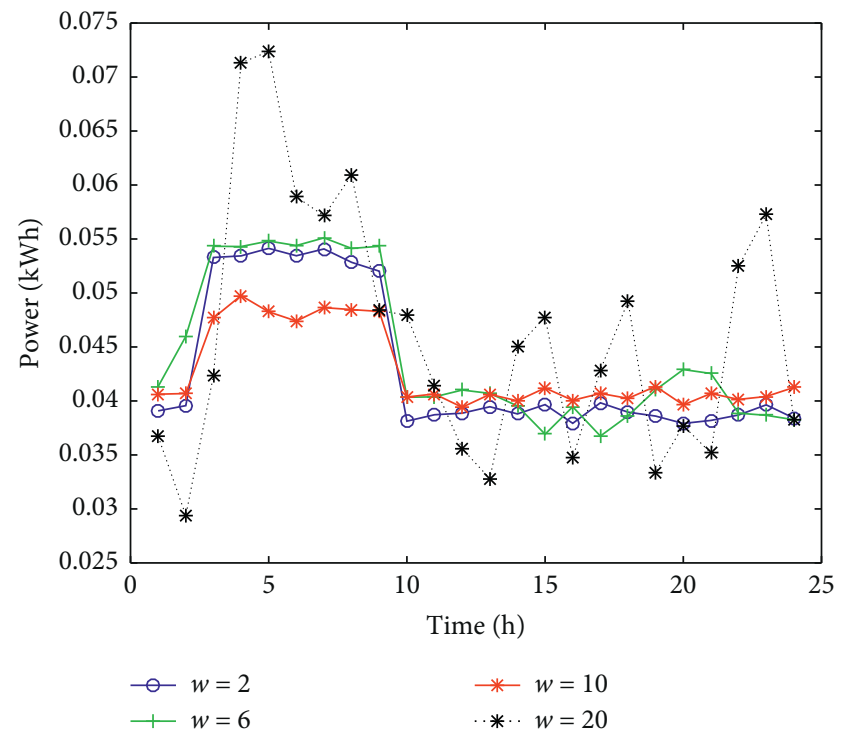

FIGURE 3: The average of electricilty consumed from the TPS by semielastic appliances $a_{7}$.



FIGURE 4: The average of electricity consumed from the TPS by semielastic appliances $a_{8}$. 


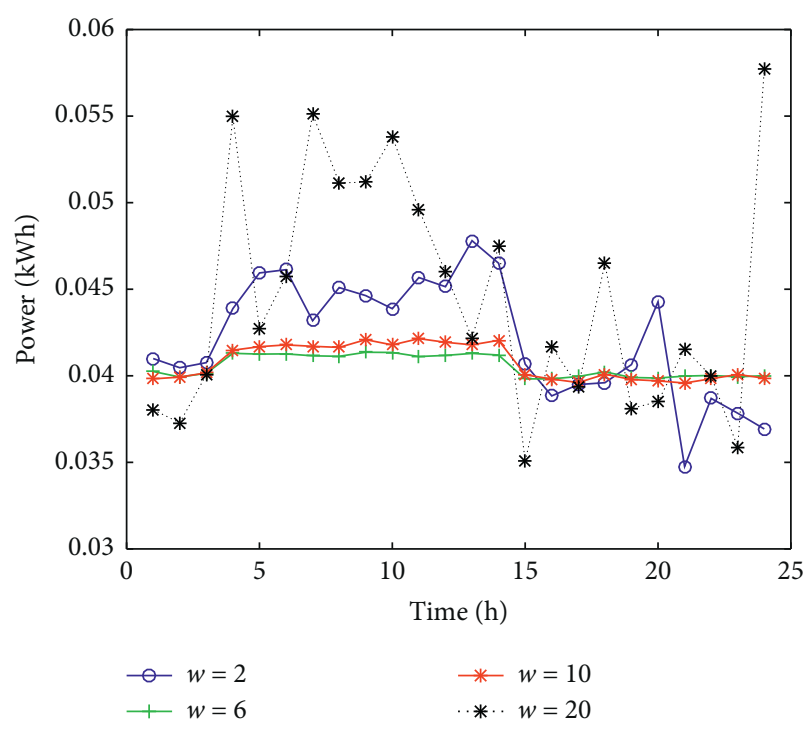

FIGURE 5: The average of electricity consumed from the TPS by semielastic appliances $a_{9}$.

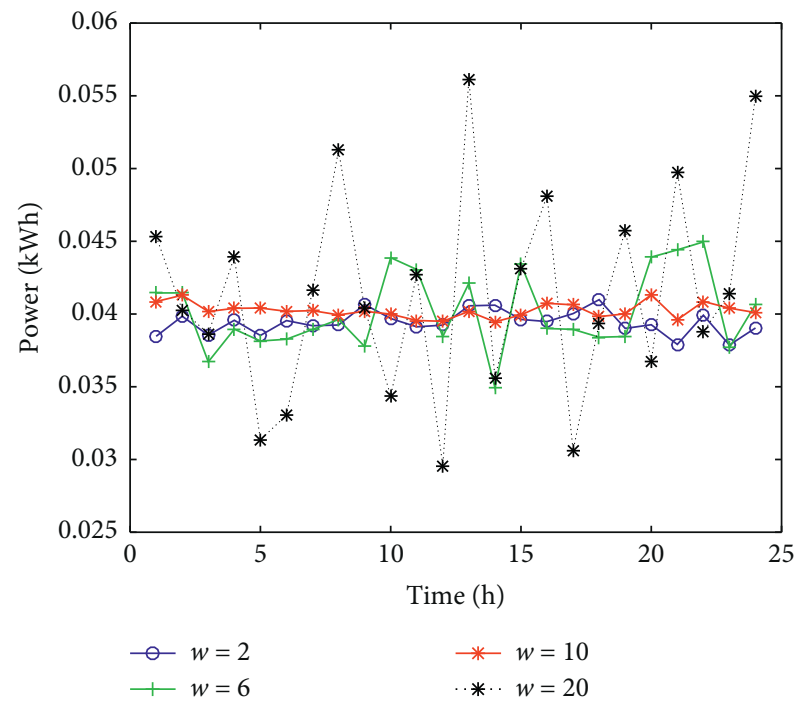

FIGURE 6: The average of electricity consumed from the TPS by semielastic appliances $a_{10}$.

4.1. Discussion under Different Elastic Coefficients. $\omega_{i}^{k}$ is selected randomly from the intervals $[1,3,5,7,9,11]$ and $[18,20]$, respectively. " $w=2$ " means $\omega_{i}^{k} \in[1,3]$ for the lowest satisfaction level; " $w=6$ " and " $w=10$ " mean $\omega_{i}^{k} \in[5,7]$ and $\omega_{i}^{k} \in[9,11]$ for the normal satisfaction levels, respectively; and " $w=20$ " means $\omega_{i}^{k} \in[19,21]$ for the highest satisfaction level. The specifications of these units are summarized in Table 6.

Through the simulations, the electricity consumption from the TPS by the semielastic appliance $a_{7}$ at each hour is computed and their average values are determined. The cycle of appliance $a_{7}$ is denoted as $\{3,4, \ldots, 9\}$ (see in

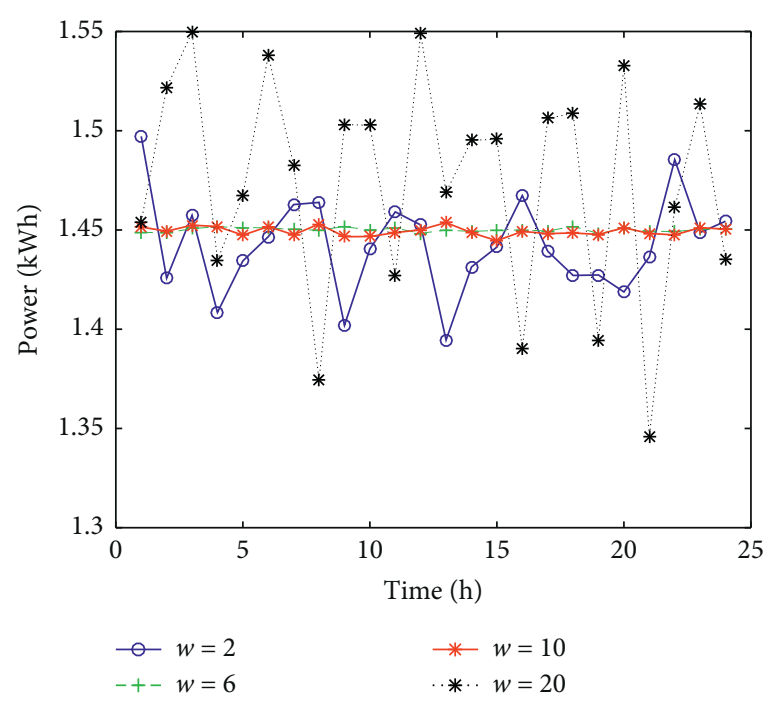

FIGURE 7: The average of electricity consumed from the TPS by elastic appliances $B_{i}$.

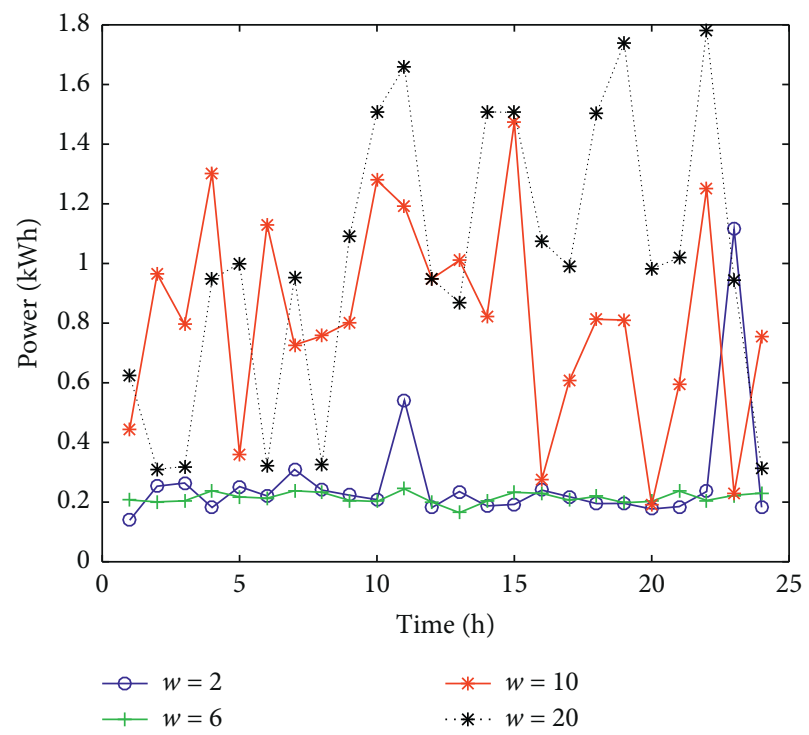

FIGURE 8: The average of electricity consumed from the RES.

Table 5) and the assignment must be completed during this period. As shown from Figure 3, the appliance $a_{7}$ is operated at time 4:00 am and is turned off at 8:00 am. Its assignment is completed within the designated time $\{3,4$, ..., 9\}. The appropriate ranges of $\omega_{i}^{k}$ in semielastic appliances are discussed, as shown from Figures 3-6. From Figure 3, when $w=2,6,10$, the electricity consumption from the TPS by scheduling the semielastic appliance $a_{7}$ is relatively smaller and stabler (relieving the supply pressure of the TPS) than that when $w=20$. The similar conclusions of other semielastic appliances are obtained, as shown from Figures $4-6$. Therefore, the value of $\omega_{i}^{k}$ under $[1,11]$ is more appropriate. 


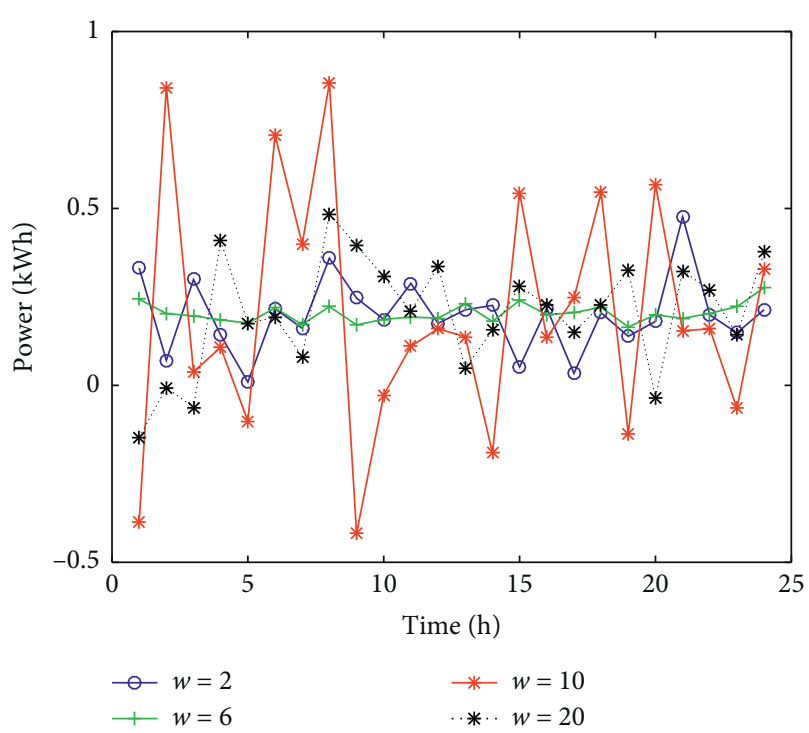

FIGURE 9: The average of electricity consumed from the ESS.

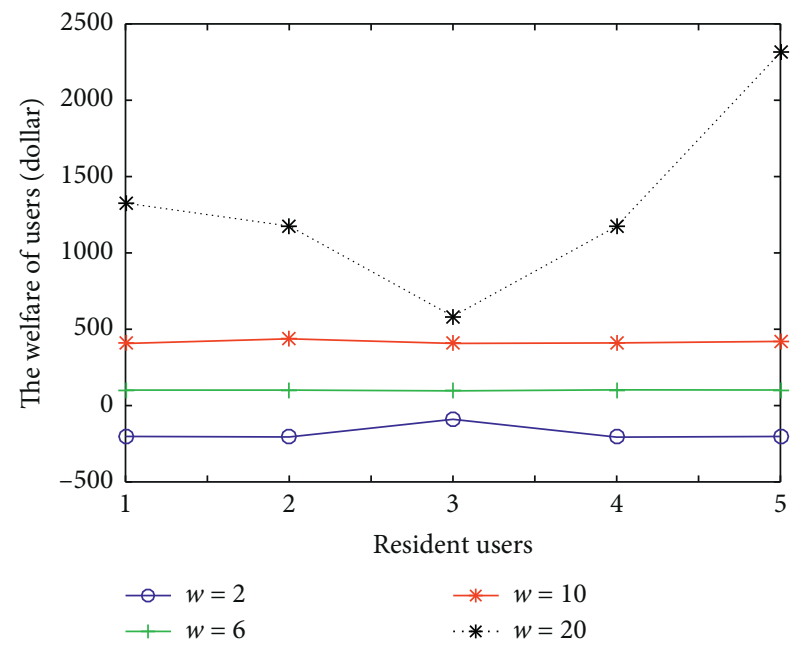

FIGURE 10: The welfare of users in four models.

TABLE 7: The indexes used in the four models.

\begin{tabular}{lccc}
\hline Model & ESS & RES & Expectation \\
\hline Allno-model & $\times$ & $\times$ & $\times$ \\
ESS-model & $\bigcirc$ & $\times$ & $\times$ \\
RES-model & $\times$ & 0 & $\bigcirc$ \\
All-model (our proposed) & $\bigcirc$ & 0 & 0 \\
\hline
\end{tabular}

Multiple simulations are conducted; the electricity consumption from the TPS by elastic appliances $B_{i}$ for five users is computed at each hour, and the average value is determined. The smaller and stabler electricity consumption from the TPS by scheduling elastic appliances is seen from, Figure 7, when $w=2$ and $w=6$. Consequently, $\omega_{i}^{k}$ is suitable under $[5,11]$ considering the influence on the TPS.

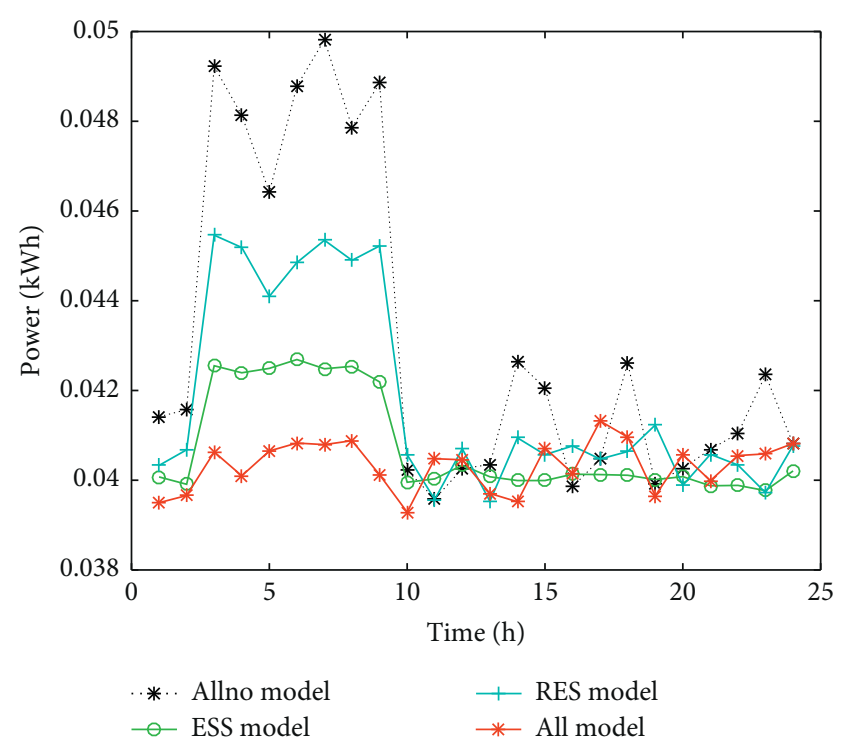

FIGURE 11: The average of electricity consumed from the TPS by semielastic appliances $a_{7}$.



FIGURE 12: The average of electricity consumed from the TPS by semielastic appliances $a_{8}$.

Users are enthusiastic for making full use of electricity consumption from the RES when $\omega_{i}^{k}$ is suitable under $[11,20]$, as shown from Figure 8 . While $w=10$, the availability of the ESS is relatively higher, especially in peak periods (10:00-20:00) discharged and off-peak periods charged, as presented in Figure 9.

As for the objective of this model, the higher the user's welfare value is when $w=20$, the worser the stability is. While not only a higher welfare value but also an optimal 


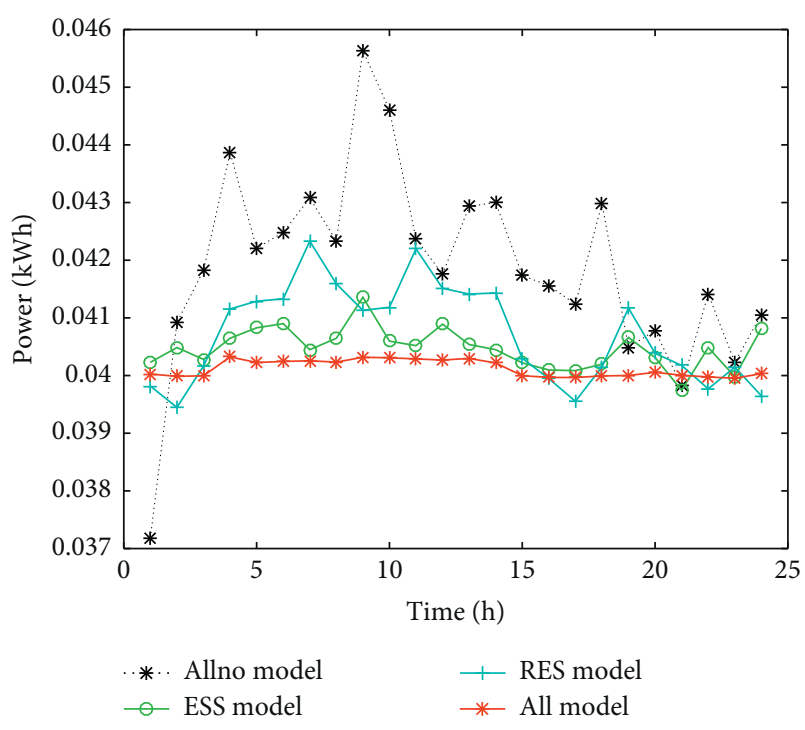

FIGURE 13: The average of electricity consumed from the TPS by semielastic appliances $a_{9}$.

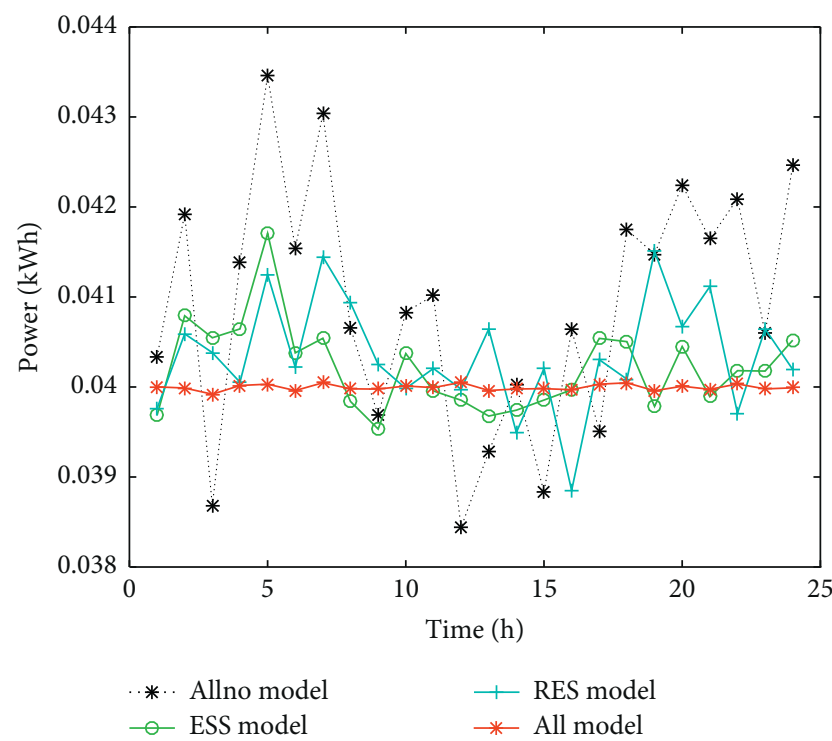

FIGURE 14: The average of electricity consumed from the TPS by semielastic appliances $a_{10}$.

solution is obtained when $w=10$, as shown in Figure 10. The users' welfare values are relatively stabler as $w=2$ and $w=6$; however, it is abnormal to get the negative values (e.g., $w=2$ ). In summary, $w=10$ (i.e., $\omega_{i}^{k} \in[9,11]$ ) is the suitable range considering both the interest of users and the influence on the TPS.

4.2. Discussion under the Same Elastic Coefficient. In this scenario, four models to illustrate the impacts of the RES and ESS by manipulating semielastic and elastic appliances are

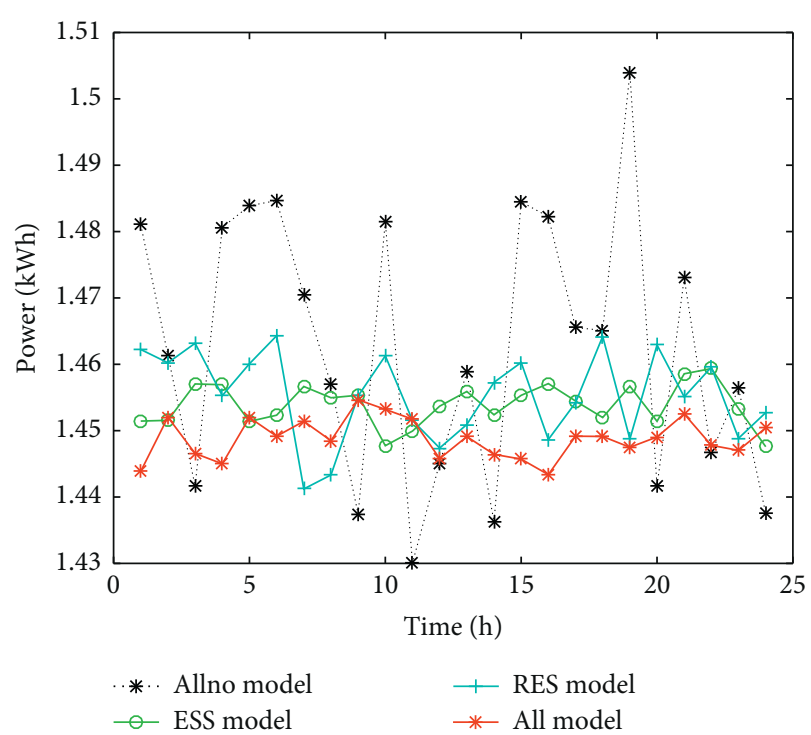

FIGURE 15: The average of electricity consumed from the TPS by elastic appliances $B_{i}$.

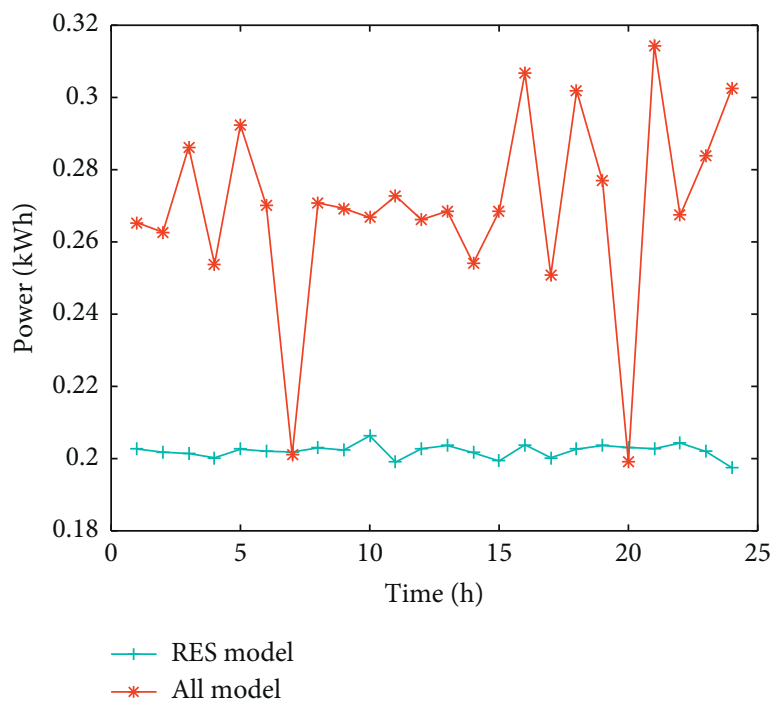

FIGURE 16: The average of electricity consumed from the RES.

studied under $\omega_{i}^{k} \in[9,11]$. The indexes used in the four models are listed in Table 7.

As can be observed from Figure 11, the average of electricity consumed from the TPS by semielastic appliances $a_{7}$ in the all-model is the least during the working periods. It is shown that the RES and ESS play positive roles as auxiliary energies making users using less power consumption from the TPS. It should be also noted, owing to the continuous working cycle of the semielastic appliances, users' aggregate power consumption (green line) from the TPS in the ESSmodel is much less than that, in the RES-model (blue line). 


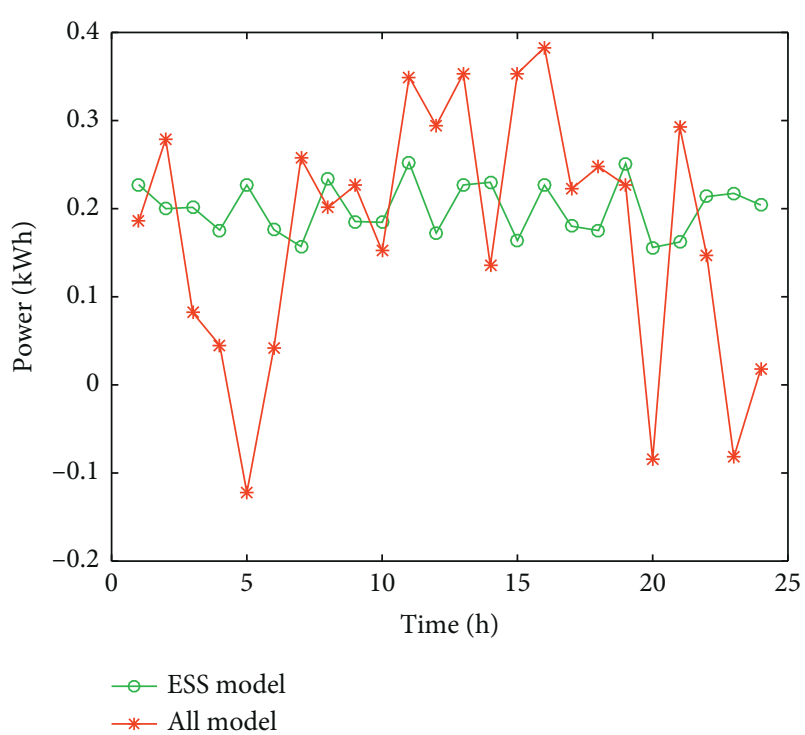

FIGURE 17: The average of electricity consumed from the ESS.



FIGURE 18: The electricity consumed from the TPS by all appliances for user 1 .

Therefore, users have priority to consume the electricity stored in the ESS and then produced from the RES and from the TPS last. By giving aggregate electricity consumption shown from Figure 11, users can reasonably arrange batteries or electrical vehicles according to their semielastic home appliances, to a large extent, alleviating the pressure of the traditional power grid. Likewise, the same conclusions are obtained from Figures 12-14.

From Figure 15, it can be seen that the electricity consumption from the TPS by elastic appliances $B_{i}$ in the allmodel is the most stable at each moment, and the range of fluctuation is the smallest of the four models. At the same time, it is observed that the ESS-model and RES-model have

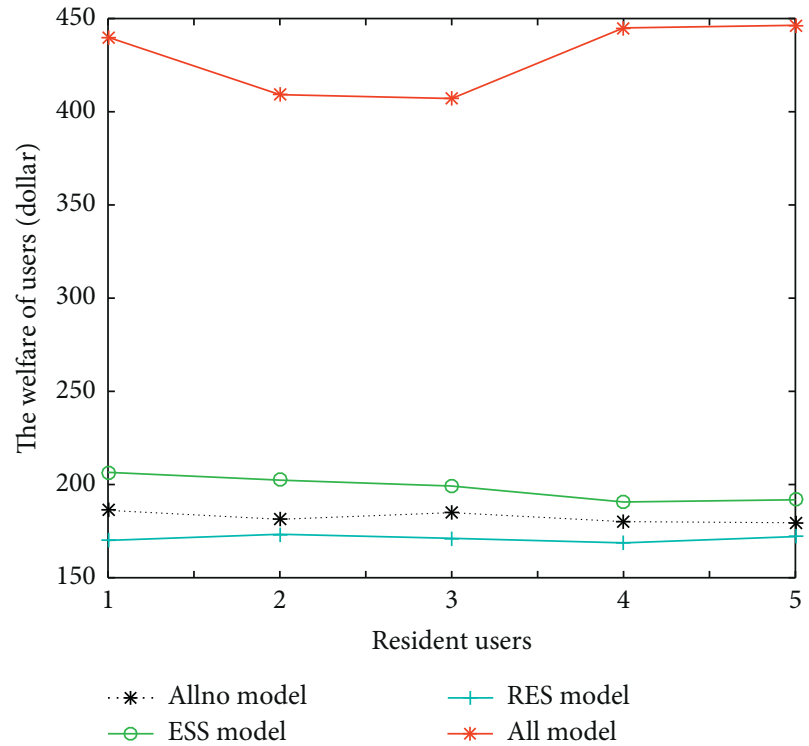

FIgURE 19: The welfare of users in four models.

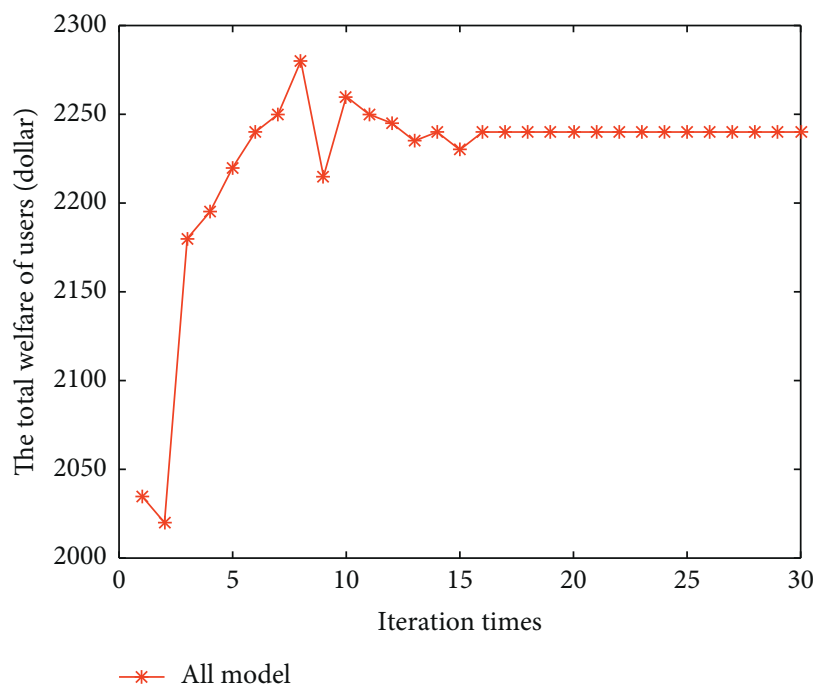

FIgURE 20: The algorithm convergence and iteration times of the all-model (our proposed model).

a similar impact in terms of electricity consumption from the TPS by elastic appliances.

From Figure 16, it can be demonstrated that the electricity consumption from the RES in the all-model is relatively higher during peak periods than that in the RESmodel, which indicates that users make full use of the RES in our proposed model. It can be seen from Figure 17 that the ESS is in the discharge states (positive values) during peak periods while in the charge states (negative values) during off-peak periods in the all-model. To some extent, the ESS relieves the power pressure on the TPS.

Without loss of generality, we consider the difference in electricity consumed from the TPS by three types of 
appliances for user 1 at each hour in four models. Figure 18 shows the electricity consumed from the TPS by all appliances for user 1 at each hour. The electricity consumption from the TPS in our proposed model is much less than those other models overall.

Figure 19 shows that the users' welfare value in the allmodel (i.e., our proposed model) is significantly higher than that of other models. Users' welfare values in the all-model are more than double those of other models under $\omega_{i}^{k} \in[9,11]$. In particular, the welfare value of user 4 reaches 448 dollars in the all-model, while it reaches 175 dollars, 180 dollars, and 200 dollars in other models, respectively. This verifies the rationality of our proposed model and feasibility of the method.

It can be demonstrated from Figure 20 that the proposed algorithm has remarkable convergence performance, and it converges at 16 times keeping the total welfare of users around 2250 dollars.

\section{Conclusions}

In this paper, an optimal residential energy consumption model is proposed, which aims to achieve a trade-off between maximizing users' utility and minimizing their payment based on scheduling household appliances. The optimal solution of the model is obtained using a smoothing quasi-Newton method. Several cases are discussed under different elastic coefficients $\omega_{i}^{k}$ which is demonstrated that $\omega_{i}^{k} \in[9,11]$ is the suitable range considering not only the interest of users but also the influence on the TPS. Afterwards, the impacts of the ESS and the RES are discussed in several models. The discussion results show that users' aggregate power consumption from the TPS by semielastic appliances in the ESS-model is much less than that in the RES-model. However, in terms of electric power consumed from the TPS by elastic appliances, the ESS-model and the RES-model have little difference. Finally, the simulation results show that our proposed model is superior to other models in terms of not only users' welfare but also electricity consumption from the TPS.

Future studies will be conducted to extend as follows:

(1) An optimal residential energy consumption model is proposed considering thermoelectrical load modeling of a house

(2) Similar models are solved adopting a robust approach or artificial intelligence algorithms such as machine-learning algorithm

(3) We should consider extending the proposed model to take care of other uncertainties (such as in the customer behavior, for scheduling appliances, or in real-time electricity price)

\section{Appendix}

\section{A. Transformation of the Objective Function}

The objective function is divided into two situations: at the initial time slot $t$ and at other time slots.

At the initial time slot $t$,

$$
E\left[\sum_{i=1}^{n}\left(U\left(l_{i}^{t}, \omega_{i}^{t}\right)-P\left(l_{i}^{t}\right)\right)\right]=\sum_{i=1}^{n}\left(U\left(l_{i}^{t}, \omega_{i}^{t}\right)-p_{t} l_{i}^{t}\right) .
$$

At other time slots,

$$
\begin{aligned}
& E\left[\sum_{k=t+1}^{K} \sum_{i=1}^{n}\left(U\left(l_{i}^{k}, \omega_{i}^{k}\right)-P\left(l_{i}^{k}\right)\right)\right] \\
& =\sum_{k=t+1}^{K} E\left[\sum_{i=1}^{n}\left(U\left(l_{i}^{k}, \omega_{i}^{k}\right)-P\left(l_{i}^{k}\right)\right)\right] .
\end{aligned}
$$

We denote

$$
V_{k}=E\left[\sum_{i=1}^{n}\left(U\left(l_{i}^{k}, \omega_{i}^{k}\right)-P\left(l_{i}^{k}\right)\right)\right] .
$$

According to the properties of the mathematical expectation, $V_{k}$ can be rewritten as follows:

$$
\begin{aligned}
V_{k} & =\sum_{i=1}^{n} E\left(U\left(l_{i}^{k}, \omega_{i}^{k}\right)-P\left(l_{i}^{k}\right)\right) \\
& =\sum_{i=1}^{n} E\left(U\left(l_{i}^{k}, \omega_{i}^{k}\right)\right)-\sum_{i=1}^{n} E\left(P\left(l_{i}^{k}\right)\right) .
\end{aligned}
$$

During operation of the power grid, power consumption of users is not saturated. Thus, only the situation $0 \leq l_{i}^{k} \leq\left(\omega_{i}^{k} / \alpha_{i}\right)$ is considered. At this time, the utility function can be written as follows:

$$
U\left(l_{i}^{k}, \omega_{i}^{k}\right)=\omega_{i}^{k} l_{i}^{k}-\frac{\alpha_{i}}{2}\left(l_{i}^{k}\right)^{2} .
$$

Then,

$$
\begin{aligned}
E\left[U\left(l_{i}^{k}, \omega_{i}^{k}\right)\right] & =E\left[\omega_{i}^{k} l_{i}^{k}-\frac{\alpha_{i}}{2}\left(l_{i}^{k}\right)^{2}\right] \\
& =\omega_{i}^{k} E\left(l_{i}^{k}\right)-\frac{\alpha_{i}}{2} E\left(l_{i}^{k}\right)^{2} .
\end{aligned}
$$

Substituting (10a) into (A.6), it is obtained that 


$$
\begin{aligned}
E\left[U\left(l_{i}^{k}, \omega_{i}^{k}\right)\right] & =\omega_{i}^{k} E\left(x_{i}^{k}-y_{i}^{t}-\sum_{j=t+1}^{k} \rho_{i}^{j}+z_{i}^{k}\right)-\frac{\alpha_{i}}{2} E\left[\left(x_{i}^{k}-y_{i}^{t}-\sum_{j=t+1}^{k} \rho_{i}^{j}+z_{i}^{k}\right)^{2}\right] \\
& =\omega_{i}^{k}\left(x_{i}^{k}-y_{i}^{t}+z_{i}^{k}\right)+\omega_{i}^{k} E\left(-\sum_{j=t+1}^{k} \rho_{i}^{j}\right)-\frac{\alpha_{i}}{2} E\left[\left(\left(x_{i}^{k}-y_{i}^{t}+z_{i}^{k}\right)^{2}-2\left(x_{i}^{t}-y_{i}^{t}+z_{i}^{k}\right) \sum_{j=t+1}^{k} \rho_{i}^{j}+\left(\sum_{j=t+1}^{k} \rho_{i}^{j}\right)^{2}\right)\right] \\
& =\omega_{i}^{k}\left(x_{i}^{k}-y_{i}^{t}+z_{i}^{k}\right)-\frac{\alpha_{i}}{2}\left(x_{i}^{k}-y_{i}^{t}+z_{i}^{k}\right)^{2}-\omega_{i}^{k} \sum_{j=t+1}^{k} \mu_{i}^{j}+\alpha_{i}\left(x_{i}^{k}-y_{i}^{t}+z_{i}^{k}\right) \sum_{j=t+1}^{k} \mu_{i}^{j}-\frac{\alpha_{i}}{2}\left(\sum_{j=t+1}^{k} \mu_{i}^{j}\right)^{2}-\frac{\alpha_{i}}{2}\left(\sum_{j=t+1}^{k} \sigma_{i}^{j}\right)^{2} \\
& =\omega_{i}^{k}\left(l_{i}^{t}\right)-\frac{\alpha_{i}}{2}\left(l_{i}^{t}\right)^{2}-\omega_{i}^{k} \sum_{j=t+1}^{k} \mu_{i}^{j}+\alpha_{i}\left(l_{i}^{t}\right) \sum_{j=t+1}^{k} \mu_{i}^{j}-\frac{\alpha_{i}}{2}\left(\sum_{j=t+1}^{k} \mu_{i}^{j}\right)^{2}-\frac{\alpha_{i}}{2}\left(\sum_{j=t+1}^{k} \sigma_{i}^{j}\right)^{2} .
\end{aligned}
$$

A utility function is redefined as $\widetilde{U}\left(l_{i}^{t}, \omega_{i}^{k}\right)=E\left[U\left(l_{i}^{k}, \omega_{i}^{k}\right)\right]$, which can be determined as follows:

$$
E\left[U_{i}\left(l_{i}^{t}, \omega_{i}^{k}\right)\right]= \begin{cases}\omega_{i}^{k} l_{i}^{t}-\frac{\alpha_{i}}{2}\left(l_{i}^{t}\right)^{2}-\omega_{i}^{k} \sum_{j=t+1}^{k} \mu_{i}^{j}+\alpha_{i} l_{i}^{t} \sum_{j=t+1}^{k} \mu_{i}^{j}-\frac{\alpha_{i}}{2}\left(\sum_{j=t+1}^{k} \mu_{i}^{j}\right)^{2}-\frac{\alpha_{i}}{2}\left(\sum_{j=t+1}^{k} \sigma_{i}^{j}\right)^{2}, & 0 \leq l_{i}^{t} \leq \frac{\omega_{i}^{k}}{\alpha_{i}}, \\ \frac{\left(\omega_{i}^{k}\right)^{2}}{2 \alpha_{i}}-\omega_{i}^{k} \sum_{j=t+1}^{k} \mu_{i}^{j}+\alpha_{i} t_{i}^{t} \sum_{j=t+1}^{k} \mu_{i}^{j}-\frac{\alpha_{i}}{2}\left(\sum_{j=t+1}^{k} \mu_{i}^{j}\right)^{2}-\frac{\alpha_{i}}{2}\left(\sum_{j=t+1}^{k} \sigma_{i}^{j}\right)^{2}, & l_{i}^{t} \geq \frac{\omega_{i}^{k}}{\alpha_{i}} .\end{cases}
$$

In addition,

$$
\begin{aligned}
E\left(P\left(l_{i}^{k}\right)\right) & =E\left(p_{k} l_{i}^{k}\right)=E\left(\max \left\{m_{k} l_{i}^{k}, n_{k} l_{i}^{k}+\left(m_{k}-n_{k}\right) b_{k}\right\}\right) \\
& =\max \left\{m_{k} E\left(l_{i}^{k}\right), n_{k} E\left(l_{i}^{k}\right)+\left(m_{k}-n_{k}\right) b_{k}\right\} \\
& =\max \left\{m_{k} E\left(\left(x_{i}^{k}-y_{i}^{k}+z_{i}^{k}\right)^{+}\right), n_{k} E\left(\left(x_{i}^{k}-y_{i}^{k}+z_{i}^{k}\right)^{+}\right)+\left(m_{k}-n_{k}\right) b_{k}\right\} \\
& =\max \left\{m_{k} E\left(\left(x_{i}^{k}-y_{i}^{t}-\sum_{j=t+1}^{k} \rho_{i}^{j}+z_{i}^{k}\right)^{+}\right), n_{k} E\left(\left(x_{i}^{k}-y_{i}^{t}-\sum_{j=t+1}^{k} \rho_{i}^{j}+z_{i}^{k}\right)^{+}\right)+\left(m_{k}-n_{k}\right) b_{k}\right\} \\
& =\max \left\{m_{k}\left(x_{i}^{k}-y_{i}^{t}-\sum_{j=t+1}^{k} \mu_{i}^{j}+z_{i}^{k}\right)^{+}, n_{k}\left(x_{i}^{k}-y_{i}^{t}-\sum_{j=t+1}^{k} \mu_{i}^{j}+z_{i}^{k}\right)^{+}+\left(m_{k}-n_{k}\right) b_{k}\right\} \\
& =\max \left\{m_{k}\left(l_{i}^{t}-\sum_{j=t+1}^{k} \mu_{i}^{j}\right)^{+}, n_{k}\left(l_{i}^{t}-\sum_{j=t+1}^{k} \mu_{i}^{j}\right)^{+}+\left(m_{k}-n_{k}\right) b_{k}\right\},
\end{aligned}
$$

where $\rho_{i}^{j}$ describes the uncertain powers produced from the RES. The expected value of distribution is $E\left(\rho_{i}^{j}\right)=\mu_{i}^{j}$, and the variance is $D\left(\rho_{i}^{j+1}\right)=\left(\sigma_{i}^{j}\right)^{2}$. By applying the probability theory, the payment function with expectation is transformed into a certain function. Therefore, a new payment function is denoted as follows: 


$$
\begin{aligned}
\widetilde{P}\left(l_{i}^{k}\right)= & E\left[P\left(l_{i}^{k}\right)\right] \\
= & \max \left\{m_{k}\left(l_{i}^{t}-\sum_{j=t+1}^{k} \mu_{i}^{j}\right)^{+}, n_{k}\left(l_{i}^{t}-\sum_{j=t+1}^{k} \mu_{i}^{j}\right)^{+}\right. \\
& \left.+\left(m_{k}-n_{k}\right) b_{k}\right\} .
\end{aligned}
$$

Substituting $\widetilde{U}\left(l_{i}^{t}, \omega_{i}^{k}\right)$ and $\widetilde{P}\left(l_{i}^{k}\right)$ into (A.6), we have

$$
\begin{aligned}
E\left[\sum_{k=t+1}^{K} \sum_{i=1}^{n}\left(U\left(l_{i}^{k}, \omega_{i}^{k}\right)-P\left(l_{i}^{k}\right)\right)\right] \\
=\sum_{k=t+1}^{K}\left[\sum_{i=1}^{n}\left(\widetilde{U}\left(l_{i}^{t}, \omega_{i}^{k}\right)-\widetilde{P}\left(l_{i}^{k}\right)\right)\right] .
\end{aligned}
$$

Considering the first case $k=t$, it can be seen that the objective function still satisfies formula (A.11). Thus, objective function (P1) can be equivalent to the following deterministic problem:

$$
\widetilde{V}=\max \sum_{k=t}^{K}\left[\sum_{i=1}^{n}\left(\widetilde{U}\left(l_{i}^{t}, \omega_{i}^{k}\right)-\widetilde{P}\left(l_{i}^{k}\right)\right)\right] .
$$

\section{B. Transformation of Constraint (9h)}

Constraint (9h) provides a probabilistic constraint on the total electricity consumption. In other words, the power supplier needs to ensure that the probability of the difference between the electricity power consumption of all users and the output of the power supplier exceeding a given threshold should be smaller than any small positive number. Thus, condition (9h) can be drastically expressed as follows:

$$
\operatorname{Pr}\left[\sum_{i=1}^{n}\left(x_{i}^{k}-y_{i}^{k}+z_{i}^{k}\right)^{+}-G_{k} \geq \eta\right] \leq \varepsilon,
$$

where $\eta$ is a given threshold, reflecting the degree of difference between the total electricity consumption and the quantity of electricity produced by the power supplier and $\varepsilon$ is a very small positive number, which can be understood as the failure probability. By replacing formula (10a) into (B.1), it can be obtained as follows:

$$
\operatorname{Pr}\left[\sum_{i=1}^{n}\left(x_{i}^{k}-\left(y_{i}^{t}+\sum_{j=t+1}^{k} \rho_{i}^{j}\right)+z_{i}^{k}\right)^{+}-G_{k} \geq \eta\right] \leq \operatorname{Pr}\left[\sum_{i=1}^{n}\left(x_{i}^{k}-y_{i}^{t}+z_{i}^{k}\right)^{+}-\sum_{i=1}^{n}\left(\sum_{j=t+1}^{k} \rho_{i}^{j}\right)-G_{k} \geq \eta\right] .
$$

Formula (B.1) can be rewritten as the following expression:

$$
\operatorname{Pr}\left[\sum_{i=1}^{n}\left(x_{i}^{k}-y_{i}^{t}+z_{i}^{k}\right)^{+}-\sum_{i=1}^{n} \sum_{j=t+1}^{k} \rho_{i}^{j}-G_{k} \geq \eta\right]=\operatorname{Pr}\left[\frac{-\sum_{i=1}^{n} \sum_{j=t+1}^{k} \rho_{i}^{j}+\mu}{\sigma} \geq \frac{\eta+G_{k}-\sum_{i=1}^{n}\left(x_{i}^{k}-y_{i}^{t}+z_{i}^{k}\right)^{+}+\mu}{\sigma}\right] \leq \varepsilon
$$

Namely,

$$
\operatorname{Pr}\left[w \geq \frac{\eta+G_{k}-\sum_{i=1}^{n}\left(x_{i}^{k}-y_{i}^{t}+z_{i}^{k}\right)^{+}+\mu}{\sigma}\right] \leq \varepsilon
$$

where $w=-\left(\sum_{i=1}^{n} \sum_{j=t+1}^{k} \rho_{i}^{j}+\mu\right) / \sigma$. Owing to $w \sim N(0,1)$, the variable obeys a standard normal with an expectation of zero and a variance of 1. Expression (B.4) can be simplified as

$$
\operatorname{Pr}\left[w \geq \frac{\eta+G_{k}-\sum_{i=1}^{n}\left(x_{i}^{k}-y_{i}^{t}+z_{i}^{k}\right)^{+}+\mu}{\sigma}\right]=\Phi\left(\frac{\eta+G_{k}-\sum_{i=1}^{n}\left(x_{i}^{k}-y_{i}^{t}+z_{i}^{k}\right)^{+}+\mu}{\sigma}\right),
$$

where

$$
\Phi(x)=\int_{x}^{\infty} \frac{1}{\sqrt{2 \pi}} e^{-t^{2} / 2} \mathrm{~d} t .
$$

Formula (B.5) is changed to

$$
\Phi\left(\frac{\eta+G_{k}-\sum_{i=1}^{n}\left(x_{i}^{k}-y_{i}^{t}+z_{i}^{k}\right)^{+}+\mu}{\sigma}\right) \leq \varepsilon .
$$

From (B.7), it follows that 


$$
\sum_{i=1}^{n}\left(x_{i}^{k}-y_{i}^{t}+z_{i}^{k}\right)^{+} \leq G_{k}+\eta+\mu-\sigma \Phi^{-1}(\varepsilon)=G_{k}+\varphi(k),
$$

where $\varphi(k)=\eta+\mu-\sigma \Phi^{-1}(\varepsilon)$.

\section{Proof of Theorem 1}

Theorem 1. Problem (P2) is a convex optimization and has an optimal solution.

Proof. According to the property of utility function, $\widetilde{U}_{i}\left(l_{i}^{t}, \omega_{i}^{k}\right)=E\left[U\left(l_{i}^{k}, \omega_{i}^{k}\right)\right]$ is a strictly concave function, whereas the sum of the strictly concave function $\sum_{k=t}^{K} \sum_{i=1}^{n} \widetilde{U}\left(l_{i}^{t}, \omega_{i}^{k}\right)$ is still a strictly concave function.

Next, it is shown that $\widetilde{P}\left(l_{i}^{k}\right)=\max \left\{m_{k}\left(l_{i}^{t}-\right.\right.$ $\left.\left.\sum_{j=t+1}^{k} \mu_{i}^{j}\right)^{+}, n_{k}\left(l_{i}^{t}-\sum_{j=t+1}^{k} \mu_{i}^{j}\right)^{+}+\left(m_{k}-n_{k}\right) b_{k}\right\}$ is a convex function. In addition, $l_{i}^{t}-\sum_{j=t+1}^{k} \mu_{i}^{j}$ is a linear function, and thus it is convex. The bigger value of two convex functions is a convex function [49], and thus $\left(l_{i}^{t}-\sum_{j=t+1}^{k} \mu_{i}^{j}\right)^{+}$is a convex function, which is called a plus function. Then, $\widetilde{P}\left(l_{i}^{k}\right)$ is a convex function, $-\widetilde{P}\left(l_{i}^{k}\right)$ is a concave function. In addition, $\sum_{k=t}^{K} \sum_{i=1}^{n} \widetilde{P}\left(l_{i}^{k}\right)$ is a convex function obtained through the property of the convex function. Thus, $-\sum_{k=t}^{K} \sum_{i=1}^{n} \widetilde{P}\left(l_{i}^{k}\right)$ is a concave function, and thus $\sum_{k=t}^{K} n \sum_{i=1}\left[\widetilde{U}\left(l_{i}^{t}, \omega_{i}^{k}\right)-\widetilde{P}\left(l_{i}^{k}\right)\right]$ is a concave function. Therefore, according to the conclusion drawn by [50] and described in Section 4.2, the concave function $\sum_{k=t}^{K} \sum_{i=1}^{n}\left[\widetilde{U}\left(l_{i}^{t}, \omega_{i}^{k}\right)-\widetilde{P}\left(l_{i}^{k}\right)\right]$ at maximum is equivalent to the minimum of a convex function. Because the constraints of problem (P2) are linear, and the feasible domain is a convex set, problem (P2) is a convex optimization. According to the convex programming property, an optimal solution of problem (P2) exists.

\section{Proof of Theorem 2}

Theorem 1. For $\forall t>0, \psi_{t}(x)$ in formula (22) is a smooth approximation function of $\psi(x)=\max (x, 0)$.

Proof. When $x>t$ or $x<t,\left|\psi_{t}(x)-\psi(x)\right|=0$, when $-t \leq x<0$, we have $\left|\psi_{t}(x)-\psi(x)\right|=\left|x^{2} / 4 t+x / 2+t / 4-0\right|=\left|(x+t)^{2} / 4 t\right| \leq t / 4$, and when $0<x \leq t$, we obtain

$$
\left|\psi_{t}(x)-\psi(x)\right|=\left|\frac{x^{2}}{4 t}+\frac{x}{2}+\frac{t}{4}-x\right|=\left|\frac{(x-t)^{2}}{4 t}\right| \leq \frac{t}{4} .
$$

Therefore, for $\forall t>0,\left|\psi_{t}(x)-\psi(x)\right| \leq(t / 4)$, and thus $\psi_{t}(x)$ is a smooth approximation function of $\psi(x)$ [47].

\section{Abbreviations}

RES: Renewable energy sources

ESS: Energy storage system

PV: Photovoltaic

PAR: Peak-to-average ratio

EQC: Electric quantity controller

RTP: Real-time pricing

IBR: Inclining block rates
TPS: Traditional power system

CL: Communication link

PL: $\quad$ Power link

SM: Smart meter

MA: Must-run appliances

EA: Elastic appliances

SEA: Semielastic appliances

DSM: Demand side management.

\section{Data Availability}

The data used to support the findings of this study are included within the article.

\section{Conflicts of Interest}

The authors declare that there are no conflicts of interest regarding the publication of this paper.

\section{Acknowledgments}

This work was supported by the National Natural Science Foundation of China (no. 11171221).

\section{References}

[1] M. Hussain and Y. Gao, "A review of demand response in an efficient smart grid environment," The Electricity Journal, vol. 31, no. 5, pp. 55-63, 2018.

[2] H. Zhu, Y. Gao, and Y. Hou, "Real-time pricing for demand response in smart grid based on alternating direction method of multipliers," Mathematical Problem in Engineering, vol. 2018, Article ID 8760575, 10 pages, 2018.

[3] A. Kharrazi, Y. Mishra, and V. Sreeram, "Discrete-event systems supervisory control for a custom power park," IEEE Transactions on Smart Grid, vol. 10, no. 1, pp. 483-492, 2019.

[4] Y. Dai, Y. Gao, H. Gao, and H. Zhu, "Real-time pricing scheme based on Stackelberg game in smart grid with multiple power retailers," Neurocomputing, vol. 260, pp. 149-156, 2017.

[5] R. Sharifi, A. Anvari-Moghaddam, S. Hamid Fathi, J. M. Guerrero, and V. Vahidinasab, "An optimal marketoriented demand response model for price-responsive residential consumers," Energy Efficiency, vol. 12, no. 3, pp. 803-815, 2019.

[6] H. Liu, W. Wang, J. Cui, and F. Tang, "Optimal power factor regulation of dispersed wind farms under diverse load and stochastic wind conditions based on improved firefly algorithm," Mathematical Problem in Engineering, vol. 2018, Article ID 6203278, 11 pages, 2018.

[7] C.-J. Huang and P.-H. Kuo, "A short-term wind speed forecasting model by using artificial neural networks with stochastic optimization for renewable energy systems," Energies, vol. 11, no. 10, p. 2777, 2018.

[8] M. Emmanuel and R. Rayudu, "Evolution of dispatchable photovoltaic system integration with the electric power network for smart grid applications: a review," Renewable and Sustainable Energy Reviews, vol. 67, pp. 207-224, 2017.

[9] A. Kyritsis, D. Voglitsis, N. Papanikolaou et al., "Evolution of PV systems in Greece and review of applicable solutions for higher penetration levels," Renewable Energy, vol. 109, pp. 487-499, 2017. 
[10] O. Rahbari, N. Omar, Y. Firouz et al., "A novel state of charge and capacity estimation technique for electric vehicles connected to a smart grid based on inverse theory and a metaheuristic algorithm," Energy, vol. 155, pp. 1047-1058, 2018.

[11] J.-M. Clairand, J. Rodríguez-García, and C. Álvarez-Bel, "Electric vehicle charging strategy for isolated systems with high penetration of renewable generation," Energies, vol. 11, no. 11, p. $3188,2018$.

[12] R. R. Appino, J. Á. González Ordiano, R. Mikut, T. Faulwasser, and V. Hagenmeyer, "On the use of probabilistic forecasts in scheduling of renewable energy sources coupled to storages," Applied Energy, vol. 210, pp. 1207-1218, 2018.

[13] K. Wang, F. Ciucu, C. Lin, and S. H. Low, "A stochastic power network calculus for integrating renewable energy sources into the power grid," IEEE Journal on Selected Areas in Communications, vol. 30, no. 6, pp. 1037-1048, 2012.

[14] M. Shahbazitabar and H. Abdi, "A novel priority-based stochastic unit commitment considering renewable energy sources and parking lot cooperation," Energy, vol. 161, pp. 308-324, 2018.

[15] M. Wolinetz, J. Axsen, J. Peters, and C. Crawford, "Simulating the value of electric-vehicle-grid integration using a behaviourally realistic model," Nature Energy, vol. 3, no. 2, pp. 132-139, 2018.

[16] P. Chaudhary and M. Rizwan, "Energy management supporting high penetration of solar photovoltaic generation for smart grid using solar forecasts and pumped hydro storage system," Renewable Energy, vol. 118, pp. 928-946, 2018.

[17] K. Nimma, M. Al-Falahi, H. D. Nguyen, S. D. G. Jayasinghe, T. Mahmoud, and M. Negnevitsky, "Grey wolf optimizationbased optimum energy-management and battery-sizing method for grid-connected microgrids," Energies, vol. 11, no. 4 , p. $847,2018$.

[18] Q. Wang, Y. Lei, and H. Cao, "Wind power prediction based on nonlinear partial least square," Mathematical Problem in Engineering, vol. 2018, Article ID 6829274, 9 pages, 2018.

[19] X. Chen, T. Wei, and S. Hu, "Uncertainty-aware household appliance scheduling considering dynamic electricity pricing in smart home," IEEE Transactions on Smart Grid, vol. 4, no. 2, pp. 932-941, 2013.

[20] Y. Wang, B. Wang, C.-C. Chu, H. Pota, and R. Gadh, "Energy management for a commercial building microgrid with stationary and mobile battery storage," Energy and Buildings, vol. 116, pp. 141-150, 2016.

[21] S. M. Hosseini, R. Carli, and M. Dotoli, "Robust day-ahead energy scheduling of a smart residential user under uncertainty," in Proceedings of the 2019 18th European Control Conference (ECC), pp. 935-940, Napoli, Italy, June 2019.

[22] L. Tao and Y. Gao, "Real-time pricing for smart grid with distributed energy and storage: a noncooperative game method considering spatially and temporally coupled constraints," International Journal of Electrical Power \& Energy Systems, vol. 115, Article ID 105487, 2020.

[23] Y. Zheng, J. Zhao, Y. Song et al., "Optimal operation of battery energy storage system considering distribution system uncertainty," IEEE Transactions on Sustainable Energy, vol. 9, no. 3, pp. 1051-1060, 2018.

[24] A. Venzke, L. Halilbasic, U. Markovic, G. Hug, and S. Chatzivasileiadis, "Convex relaxations of chance constrained AC optimal power flow," IEEE Transactions on Power Systems, vol. 33, no. 3, pp. 2829-2841, 2018.

[25] R. J. W. Groot, J. Morren, and J. G. Slootweg, "Smart integration of distribution automation applications," in
Proceedings of the 3rd IEEE PES International Conference and Exhibition on Innovative Smart Grid Technologies, pp. 1-7, Berlin, Germany, October 2012.

[26] Z. Yang, L. Sun, J. Chen, Q. Yang, X. Chen, and K. Xing, "Profit maximization for plug-in electric taxi with uncertain future electricity prices," IEEE Transactions on Power Systems, vol. 29, no. 6, pp. 3058-3068, 2014.

[27] T. Mahmoud, Z. Y. Dong, and J. Ma, “An advanced approach for optimal wind power generation prediction intervals by using self-adaptive evolutionary extreme learning machine," Renewable Energy, vol. 126, pp. 254-269, 2018.

[28] Y. Zhang, Y. Zhao, G. Pan, and J. Zhang, "Wind speed interval prediction based on Lorenz disturbance distribution," IEEE Transactions on Sustainable Energy, vol. 11, pp. 807-816, 2020.

[29] K. Li, R. Wang, H. Lei, T. Zhang, Y. Liu, and X. Zheng, "Interval prediction of solar power using an improved Bootstrap method," Solar Energy, vol. 159, pp. 97-112, 2018.

[30] R. Deng, Z. Yang, M.-Y. Chow, and J. Chen, "A survey on demand response in smart grids: mathematical models and approaches," IEEE Transactions on Industrial Informatics, vol. 11, no. 3, pp. 570-582, 2015.

[31] B. Ke, T. Ku, Y. Ke, C. Chuang, and H. Chen, "Sizing the battery energy storage system on a university campus with prediction of load and photovoltaic generation," in Proceedings of the 51st IEEE/IAS Industrial \& Commercial Power Systems Technical Conference, pp. 1136-1147, Calgary, Canada, May 2015.

[32] T. Nakayama, K. Mineno, T. H. T. Nguyen, and M. Ishida, “A method for capacity designing of a photovoltaic power generation with a battery using annual total amount of solar radiation analysis model," in Proceedings of the 51st International Universities' Power Engineering Conference, pp. 1-6, Coimbra, Portugal, September 2016.

[33] G. Xu, W. Yu, D. Griffith, N. Golmie, and P. Moulema, "Towards integrating distributed energy resources and storage devices in smart grid," IEEE Internet of Things Journal, vol. 4, no. 1, pp. 192-204, 2016.

[34] R. S. Liu and Y. F. Hsu, "A scalable and robust approach to demand side management for smart grids with uncertain renewable power generation and bi-directional energy trading," International Journal of Electrical Power and Energy Systems, vol. 97, pp. 396-407, 2018.

[35] P. Samadi, H. Mohsenian-Rad, V. W. S. Wong, and R. Schober, "Utilizing renewable energy resources by adopting DSM techniques and storage facilities," in Proceedings of the IEEE International Conference on Communications, pp. 4221-4226, Sydney, NSW, Australia, June 2014.

[36] L. Park, Y. Jang, S. Cho, and J. Kim, "Residential demand response for renewable energy resources in smart grid systems," IEEE Transactions on Industrial Informatics, vol. 13, no. 6, pp. 3165-3173, 2017.

[37] K. Paridari, A. Parisio, H. Sandberg, and K. H. Johansson, "Robust scheduling of smart appliances in active apartments with user behavior uncertainty," IEEE Transactions on Automation Science and Engineering, vol. 13, no. 1, pp. 247-259, 2016.

[38] S. Chen, N. B. Shroff, and P. Sinha, "Heterogeneous delay tolerant task scheduling and energy management in the smart grid with renewable energy," IEEE Journal on Selected Areas in Communications, vol. 31, no. 7, pp. 1258-1267, 2013.

[39] A. Anvari-Moghaddam, H. Monsef, and A. Rahimi-Kian, "Optimal smart home energy management considering energy saving and a comfortable lifestyle," IEEE Transactions on Smart Grid, vol. 6, no. 1, pp. 324-332, 2015. 
[40] A. Anvari-Moghaddam, H. Monsef, and A. Rahimi-Kian, "Cost-effective and comfort-aware residential energy management under different pricing schemes and weather conditions," Energy and Buildings, vol. 86, pp. 782-793, 2015.

[41] A. Anvari-Moghaddam, J. M. Guerrero, A. Rahimi-Kian, and M. S. Mirian, "Optimal real-time dispatch for integrated energy systems: an ontology-based multi-agent approach," in Proceedings of the IEEE 7th International Symposium on Power Electronics for Distributed Generation Systems, pp. 1-6, Vancouver, Canada, June 2016.

[42] A. Anvari-Moghaddam, A. Rahimi-Kian, M. S. Mirian, and J. M. Guerrero, "A multi-agent based energy management solution for integrated buildings and microgrid system," Applied Energy, vol. 203, pp. 41-56, 2017.

[43] M. Geidl, G. Koeppel, P. Favre-Perrod, B. Klockl, G. Andersson, and K. Frohlich, "Energy hubs for the future," IEEE Power and Energy Magazine, vol. 5, no. 1, pp. 24-30, 2007.

[44] R. Carli and M. Dotoli, "Decentralized control for residential energy management of a smart users microgrid with renewable energy exchange," IEEE/CAA Journal of Automatica Sinica, vol. 6, no. 3, pp. 641-656, 2019.

[45] A.-H. Mohsenian-Rad and A. Leon-Garcia, "Optimal residential load control with price prediction in real-time electricity pricing environments," IEEE Transactions on Smart Grid, vol. 1, no. 2, pp. 120-133, 2010.

[46] H. Zhu, Y. Gao, Y. Hou, and L. Tao, "Multi-time slots realtime pricing strategy with power fluctuation caused by operating continuity of smart home appliances," Engineering Applications of Artificial Intelligence, vol. 71, pp. 166-174, 2018.

[47] Y. Gao, Nonsmooth Optimization, Science Press, Beijing, China, 2018.

[48] P. Samadi, A. H. Mohsenian-Rad, R. Schober, V. W. S. Wong, and J. Jatskevich, "Optimal real-time pricing algorithm based on utility maximization for smart grid," in Proceedings of the IEEE International Conference on Smart Grid, pp. 415-420, Gaithersburg, MD, USA, October 2010.

[49] L. P. Qian, Y. J. A. Zhang, J. Huang, and Y. Wu, "Demand response management via real-time electricity price control in smart grids," IEEE Journal on Selected Areas in Communications, vol. 31, no. 7, pp. 1268-1280, 2013.

[50] S. Boyd and L. Vandenberghe, Convex Optimization, Cambridge University Press, Cambridge, UK, 2004. 